\title{
The existence and multiplicity of positive solutions for a class of nonlocal elliptic problems
}

\section{Baoqiang Yan* and Tianfu Ma}

\section{"Correspondence:}

yanbqcn@aliyun.com

School of Mathematical Sciences,

Shandong Normal University, Jinan,

250014, P.R. China

\begin{abstract}
In this paper, we prove the existence of a solution between a well-ordered subsolution and supersolution of a class of nonlocal elliptic problems and give some degree information. Using the method and bifurcation theory, we present the existence and multiplicity of positive solutions for the nonlocal problems with the changes of the parameter.

MSC: $35 \mathrm{~J} 60 ; 35 \mathrm{~J} 75 ; 47 \mathrm{H} 10$

Keywords: sub-supersolution method; bifurcation theory; nonlocal elliptic equations; degree; existence; multiplicity
\end{abstract}

\section{Introduction}

In this paper, we consider the following problem:

$$
\left\{\begin{array}{l}
-a\left(\int_{\Omega}|u|^{\gamma} d x\right) \Delta u=f_{\lambda}(x, u), \quad x \text { in } \Omega, \\
u>0, \quad x \text { in } \Omega \\
u=0, \quad x \text { on } \partial \Omega,
\end{array}\right.
$$

where $\Omega \subseteq R^{N}$ is a smooth bounded domain, $\gamma \in(0,+\infty)$, and $a:[0,+\infty) \rightarrow(0,+\infty)$ is a continuous function with

$$
\inf _{t \in[0,+\infty)} a(t) \geq a_{0}=a(0)>0
$$

Chipot and Lovat [1] considered the following model problem:

$$
\left\{\begin{array}{l}
u_{t}-a\left(\int_{\Omega} u(z, t) d z\right) \Delta u=f \quad \text { in } \Omega \times(0, T) \\
u(x, t)=0 \text { on } \Gamma \times(0, T) \\
u(x, 0)=u_{0}(x) \text { on } \Omega
\end{array}\right.
$$

Here $\Omega$ is a bounded open subset in $R^{N}, N \geq 1$, with smooth boundary $\Gamma$, and $T$ is an arbitrary time. The diffusion coefficient $a$ is a function from $R$ into $(0,+\infty)$, which depends on the entire population in the domain rather than on the local density, and $u$ describes

(c) 2016 Yan and Ma. This article is distributed under the terms of the Creative Commons Attribution 4.0 International License (http://creativecommons.org/licenses/by/4.0/), which permits unrestricted use, distribution, and reproduction in any medium, provided you give appropriate credit to the original author(s) and the source, provide a link to the Creative Commons license, and indicate if changes were made. 
the density of a population subject to spreading. If $\gamma=2$, then we get the well-known Carrier equation. The fact that (1.1) appears in some applied mathematics attracts a lot of attention. With the aid of the Krasnoselskii fixed point theorem and the Schaefer fixed point theorem, by the monotonicity of $f_{\lambda}$, Corrêa [2] considered the existence of positive solutions of (1.1) for $\gamma \geq 1$. By establishing a comparison principle, Corrêa et al. [3] proved the existence of positive solutions to (1.1) also for $\gamma=1$. Under the assumption that $A(x, u)$ (which is generalized from the nonlocal term $a(s)$ ) is bounded, there are some results on the existence of positive solutions and the existence of $n$ distinct solutions; see $[4,5]$.

Another nonlocal elliptic equations are the Kirchhoff elliptic problems like

$$
\left\{\begin{array}{l}
-a\left(\|u\|^{2}\right) \Delta u=f_{\lambda}(x, u), \quad x \text { in } \Omega, \\
u>0, \quad x \text { in } \Omega, \\
u=0, \quad x \text { on } \partial \Omega,
\end{array}\right.
$$

related to nonlinear vibrations of beams, where $a: R \rightarrow R$ is a given function, and $\|\cdot\|$ denotes the usual norm in $H_{0}^{1}(\Omega)$. In this case, variational methods are used to consider the existence of the solutions to (1.2) because the nonlocal operator $u \rightarrow a(\|u\|) \Delta u$ possesses a variational structure; see [6-13] and the references therein. Especially, for the Kirchhoff elliptic equations

$$
\left\{\begin{array}{l}
-a\left(\|u\|^{2}\right) \Delta u=\lambda f(x)|u|^{q-2} u+g(x)|u|^{p-2} u, \quad x \text { in } \Omega \\
u>0, \quad x \text { in } \Omega \\
u=0, \quad x \text { on } \partial \Omega
\end{array}\right.
$$

Chen et al. [14] examined in detail the number of solutions admitted subject to the variations of parameters embedded in nonlinear terms. For the case $a(t) \equiv 1$, the existence and multiplicity of positive solutions for the elliptic equations has been extensively investigated; see [15-22]. Especially, Ambrosetti et al. [23] studied the equation

$$
\left\{\begin{array}{l}
-\Delta u=\lambda u^{q}+u^{p}, \quad x \text { in } \Omega, \\
u>0, \quad x \text { in } \Omega, \\
u=0, \quad x \text { on } \partial \Omega,
\end{array}\right.
$$

and established multiple results for different $\lambda$, where $\Omega$ is a bounded domain in $R^{N}$ with $0<q<1<p \leq 2^{*}\left(2^{*}=\frac{2 N}{N-2}\right.$ if $N \geq 3$ and $2^{*}=+\infty$ if $\left.N=1,2\right)$ and $\lambda>0$.

Naturally, we hope that there are some interesting results for (1.1) that are similar to those in (1.3) and (1.4) in [14,23] and references therein. Notice that the methods used in $[8-11,14-16,19,21-25]$ are the sub-suppersolution method, theory of topological degree, and the variational method. Unfortunately, the operator $u \rightarrow a\left(\int_{\Omega}|u(x)|^{\gamma} d x\right) \Delta u$ has no variational structure. Up to now, the tools to study (1.1) are a fixed point result with LeraySchauder condition and the Schaefer fixed point theorem. Very recently, Alves and Covei [26] established the sub-supersolution method, which can be used to study the existence of weak solutions for a large class of nonlocal problems.

Motivated by the works of $[14,23,26,27]$, in this paper, we study the existence and multiplicity of the classical positive solutions for (1.1). The paper is organized as follows. In Section 2, according to the idea in $[26,28]$, we prove the existence of solutions be- 
tween well-ordered subsolution and supersolution to guarantee the existence of classical solution to (2.1) and give a formula to calculate the degree. Section 3 presents the existence and multiplicity of positive solutions to $(3.1)_{\lambda}$ when $p>1>q>0$ or $1>p>q>0$, which improves the results in [29], where the $a(t)$ is bounded, or $\Omega$ is an annular region. In Section 4 , when nonlinearity is linear at $u=0$, by bifurcation theory we discuss the unbounded connected component for $(4.1)_{\lambda}$ and present sufficient and necessary conditions for the existence of positive solutions to (4.7) ${ }_{\lambda}$. In Section 5, in the case where the nonlinear term is singular at $u=0$, we consider the existence of positive solutions to $(5.1)_{\lambda}$. In Section 6, sufficient and necessary conditions of positive solutions to (5.1) are given to guarantee that positive solutions to $(5.1)$ are in $C[0,1]$ or $C^{1}[0,1]$ when $N=1$.

Notation In this paper we use the following notation.

Let $u: \bar{\Omega} \rightarrow R$ is continuous, and $|u|_{\infty}=\max _{x \in \bar{\Omega}}|u(x)|$;

$C(\bar{\Omega})=\{u: \bar{\Omega} \rightarrow R \mid u(x)$ is continuous on $\bar{\Omega}\}$ with norm $\|u\|=|u|_{\infty}$;

$C^{1}(\bar{\Omega})=\{u \in C(\bar{\Omega}) \mid \nabla u(x)$ is continuous on $\bar{\Omega}\}$ with norm $\|u\|=\max \left\{|u|_{\infty},|\nabla u|_{\infty}\right\}$.

\section{Sub-supersolution method}

Now we consider the general problem

$$
\left\{\begin{array}{l}
-a\left(\int_{\Omega}|u|^{\gamma} d x\right) \Delta u=F(x, u), \quad x \text { in } \Omega \\
u=0, \quad x \text { on } \partial \Omega
\end{array}\right.
$$

where $\Omega \subseteq R^{N}$ is a smooth bounded domain, $\gamma \in(0,+\infty)$, and $a:[0,+\infty) \rightarrow(0,+\infty)$ is a continuous function with

$$
a_{0}=a(0)>0
$$

Definition 2.1 The pair functions $\alpha, \beta \in C^{1}(\bar{\Omega}) \cap C^{2}(\Omega)$ are subsolution and supersolution of (2.1) if

$$
\left\{\begin{array}{l}
-\Delta \alpha(x) \leq \frac{1}{a\left(\left.\int_{\Omega}|x(x, u(x))|\right|^{\gamma} d x\right)} F(x, \alpha(x)), \quad x \text { in } \Omega, \forall u \in C^{1}(\bar{\Omega}) \cap C^{2}(\Omega), \\
\left.\alpha\right|_{\partial \Omega} \leq 0
\end{array}\right.
$$

and

$$
\left\{\begin{array}{l}
-\Delta \beta(x) \geq \frac{1}{a\left(\int_{\Omega}|x(x, u(x))|^{\gamma} d x\right)} F(x, \beta(x)), \quad x \text { in } \Omega, \forall u \in C^{1}(\bar{\Omega}) \cap C^{2}(\Omega), \\
\left.\beta\right|_{\partial \Omega} \geq 0
\end{array}\right.
$$

where $\chi(x, u)=\alpha(x)+(u-\alpha(x))^{+}-(u-\beta(x))^{+}$.

Definition 2.2 Let $u, v \in C^{1}(\bar{\Omega})$. We say that $u \prec v$ if $u(x)<v(x)$ on $\Omega$ and $u(x) \leq v(x)$ for all $x \in \partial \Omega$, and if $u(x)=v(x)$ for some $x \in \Gamma \subseteq \partial \Omega$, then we write $\left.\frac{\partial u}{\partial n}\right|_{x \in \partial \Gamma}>\left.\frac{\partial v}{\partial n}\right|_{x \in \Gamma}$.

Remark 2.1 $S=\left\{u \in C^{1}(\bar{\Omega}): \alpha \prec u \prec \beta\right\}$ is an open set if $\alpha \prec \beta$.

We say that an open set $S \subseteq C^{1}(\bar{\Omega})$ is admissible for the degree if the compact operator $A$ has no fixed point on its boundary $\partial S$ and the set of fixed points of $A$ in $S$ is bounded. 
In this case, we define

$$
\operatorname{deg}(I-A, S, \theta)=\operatorname{deg}(I-A, S \cap B(0, R), \theta),
$$

where $R$ is such that every fixed point $u$ of $A$ in $S$ satisfies $\|u\|<R$. By excision property this degree does not depend on $R$.

To be able to associate a degree with a pair of subsolution and supersolution, we have to reinforce the definition.

Definition 2.3 A subsolution $\alpha$ of (2.1) is said to be strict if every solution $u$ of (2.1) such that $\alpha \leq u$ satisfies $\alpha \prec u$.

In the same way, a strict supersolution $\beta$ of (2.1) is a supersolution such that every solution $u$ of (2.1) such that $u \leq \beta$ satisfies $u \prec \beta$.

Definition 2.4 The function $F: \Omega \times R$ is an $L^{p}$-Carathéodory function if

1. $F(\cdot, u)$ is measurable for all $u \in \Omega$;

2. $F(x, \cdot)$ is continuous for a.e. $x \in \Omega$;

3. for all bounded set $B \subseteq R^{N}$, there exists $h_{B} \in L^{p}(\Omega)$ such that for a.e. $x \in \Omega$ and all $u \in B$,

$$
|F(x, u)| \leq h_{B}(x)
$$

Remark 2.2 The idea of the above definitions comes from [28].

If $F$ is an $L^{p}$-Carathéodory function with $p>N$, then the operator

$$
N: C^{1}(\bar{\Omega}) \rightarrow L^{p}(\Omega): u \mapsto \frac{F(x, u(x))}{a\left(\int_{\Omega}|u(x)|^{\gamma} d x\right)}
$$

is well defined, continuous, and maps bounded sets to bounded sets. Then the operator $A: C^{1}(\bar{\Omega}) \rightarrow C^{1}(\bar{\Omega})$ defined as

$$
A u=(-\Delta+\lambda)^{-1}(N u+\lambda u), \quad \lambda>0,
$$

is completely continuous, and problem (2.1) is

$$
u=A u, \quad \lambda>0 .
$$

Theorem 2.1 Let $\Omega \subseteq R^{N}(N \geq 1)$ be a smooth bounded domain, and $\gamma \in(0,+\infty)$. Suppose that $F: \Omega \times R \rightarrow R$ is a continuous function. Assume that $\alpha$ and $\beta$ are the subsolution and supersolution of (2.1), respectively. If there exists $h \in L^{p}(\Omega)(p>N)$ such that

$$
|F(x, u)| \leq h(x), \quad x \in \Omega, \alpha(x) \leq u \leq \beta(x) .
$$

Then problem (2.1) has at least one solution $u$ such that, for all $x \in \bar{\Omega}$,

$$
\alpha(x) \leq u(x) \leq \beta(x) .
$$


If, moreover, $\alpha(x)$ and $\beta(x)$ are strict and satisfy $\alpha \prec \beta$, then

$$
S=\left\{u \in C^{1}(\bar{\Omega}) \mid \alpha \prec \beta\right\}
$$

is admissible for the degree, and

$$
\operatorname{deg}(I-A, S, \theta)=1 .
$$

Proof By $L^{p}$-theory there exists $R>0$ greater than $\max \{\|\alpha\|,\|\beta\|\}$ such that, for every $F$ satisfying (2.2) and every solution of (2.1) with $\alpha \leq u \leq \beta$, we have

$$
\|u\|<R
$$

Let

$$
\bar{F}(x, u)= \begin{cases}F(x, \alpha(x)) & \text { if } u<\alpha(x) \\ F(x, u) & \text { if } \alpha(x) \leq u \leq \beta(x) \\ F(x, \beta(x)) & \text { if } u>\beta(x)\end{cases}
$$

We will study the modified problem $(\lambda>0)$

$$
\left\{\begin{array}{l}
-\Delta u+\lambda u=\frac{\bar{F}(x, u)}{a\left(\int_{\Omega}|\chi(x, u(x))|^{\gamma} d x\right)}+\lambda \chi(x, u), \quad x \in \Omega, \\
\left.u\right|_{\partial \Omega}=0
\end{array}\right.
$$

where $\chi(x, u)=\alpha(x)+(u-\alpha(x))^{+}-(u-\beta(x))^{+}$.

Step 1. Every solution $u$ of (2.3) is such that $\alpha(x) \leq u(x) \leq \beta(x), x \in \bar{\Omega}$.

We prove that $\alpha(x) \leq u(x)$ on $\bar{\Omega}$. By contradiction assume that $\max _{x \in \bar{\Omega}}(\alpha(x)-u(x))=$ $M>0$. Note that $\alpha(x)-u(x) \not \equiv M$ on $\bar{\Omega}(\alpha(x)-u(x) \leq 0, x \in \partial \Omega)$. If $x_{0} \in \Omega$ is such that $\alpha\left(x_{0}\right)-u\left(x_{0}\right)=M$, then

$$
\begin{aligned}
0 \leq & -\Delta\left(\alpha\left(x_{0}\right)-u\left(x_{0}\right)\right) \\
\leq & \frac{1}{a\left(\int_{\Omega}\left|\chi\left(x, u_{0}(x)\right)\right|^{\gamma} d x\right)} F\left(x_{0}, \alpha\left(x_{0}\right)\right) \\
& -\frac{1}{a\left(\int_{\Omega}\left|\chi\left(x, u_{0}(x)\right)\right|^{\gamma} d x\right)} \bar{F}\left(x_{0}, u\left(x_{0}\right)\right)-\lambda \chi\left(x_{0}, u\left(x_{0}\right)\right)+\lambda u\left(x_{0}\right) \\
= & -\lambda\left(\alpha\left(x_{0}\right)-u\left(x_{0}\right)\right) \\
< & 0 .
\end{aligned}
$$

This is a contradiction.

Now we prove that $\beta(x) \geq u(x)$ on $\bar{\Omega}$. By contradiction assume that $\max _{x \in \bar{\Omega}}(\beta-u(x))=$ $-m<0$. Note that $\beta(x)-u(x) \not \equiv-m$ on $\bar{\Omega}(\beta(x)-u(x) \geq 0, x \in \partial \Omega)$. If $x_{0} \in \Omega$ is such that: $\beta\left(x_{0}\right)-u\left(x_{0}\right)=-m$, then

$$
\begin{aligned}
0 & \geq-\Delta\left(\beta\left(x_{0}\right)-u\left(x_{0}\right)\right) \\
& \geq \frac{1}{a\left(\int_{\Omega}\left|\chi\left(x, u_{0}(x)\right)\right|^{\gamma} d x\right)} F\left(x_{0}, \beta\left(x_{0}\right)\right)
\end{aligned}
$$




$$
\begin{aligned}
& -\frac{1}{a\left(\int_{\Omega}\left|\chi\left(x, u_{0}(x)\right)\right|^{\gamma} d x\right)} \bar{F}\left(x_{0}, u\left(x_{0}\right)\right)-\lambda \chi\left(x_{0}, u\left(x_{0}\right)\right)+\lambda u\left(x_{0}\right) \\
= & \lambda\left(\beta\left(x_{0}\right)-u\left(x_{0}\right)\right) \\
> & 0 .
\end{aligned}
$$

This is a contradiction.

Consequently,

$$
\alpha(x) \leq u(x) \leq \beta(x), \quad x \in \Omega .
$$

Step 3. Every solution of (2.3) is a solution of (2.1). Every solution of (2.3) is such that $\alpha(x) \leq u(x) \leq \beta(x)$. Since $\bar{F}$ satisfies (2.2), we also have that $\|u\|<R$. Hence,

$$
\bar{F}(x, u(x))=F(x, u(x)), \quad \frac{1}{a\left(\int_{\Omega}|\chi(x, u(x))|^{\gamma} d x\right)}=\frac{1}{a\left(\int_{\Omega}|u(x)|^{\gamma} d x\right)},
$$

and $u$ is a solution of (2.1).

Step 4. Problem (2.3) has at least one solution.

Define the operator

$$
\bar{N}: C^{1}(\bar{\Omega}) \rightarrow L^{p}(\Omega): u \mapsto \frac{\bar{F}(x, u(x))}{a\left(\int_{\Omega}|u(x)|^{\gamma} d x\right)} .
$$

It is easy to see that $\bar{N}$ is well defined, continuous, and maps bounded sets to bounded sets. Then the operator $\bar{A}: C^{1}(\bar{\Omega}) \rightarrow C^{1}(\bar{\Omega})$ defined as

$$
\bar{A} u=(-\Delta+\lambda)^{-1}(\bar{N} u+\lambda u)
$$

is completely continuous.

By the hypothesis on $F$ and the construction of $\bar{F}$ there exists $h \in L^{p}(\Omega)$ such that, for every $u \in C^{1}(\bar{\Omega})$,

$$
\left|\frac{\bar{F}(x, u(x))}{a\left(\int_{\Omega}|\chi(x, u(x))|^{\gamma} d x\right)}+\lambda \chi(x, u(x))\right|<h(x),
$$

which guarantees that there exists $K>0$ large enough such that, for all $v \in \bar{A}\left(C^{1}(\bar{\Omega})\right)$,

$$
\|v\| \leq K
$$

Then there exists $\bar{K}>\max \{\|\alpha\|,\|\beta\|\}$ large enough such that

$$
\bar{A}\left(\overline{B_{C^{1}}(0, \bar{K})}\right) \subseteq B_{C^{1}}(0, \bar{K})
$$

and, by a classical result of degree theory [30],

$$
\operatorname{deg}\left(I-\bar{A}, B_{C^{1}}(0, \bar{K}), \theta\right)=1 .
$$


Therefore, there exists $u \in B_{C^{1}}(0, \bar{K})$ such that

$$
u=\bar{A} u .
$$

Steps 2 and 3 yield that

$$
\alpha(x) \leq u(x) \leq \beta(x), \quad x \in \Omega .
$$

Step 5. If $\alpha(x)$ and $\beta(x)$ are the strict subsolution and supersolution, then we have

$$
\operatorname{deg}(I-A, S, \theta)=1 .
$$

Since $\alpha(x)$ and $\beta(x)$ are the strict subsolution and supersolution, $A$ has no fixed point on $\partial S$, and so $\operatorname{deg}(I-A, S, \theta)$ is well defined. Step 6 guarantees that $\bar{A}$ has no fixed point in $B(0, \bar{K})-\bar{S}$. Then

$$
\operatorname{deg}(I-A, S, \theta)=\operatorname{deg}\left(I-\bar{A}, B_{C^{1}}(0, \bar{K}), \theta\right)=1 .
$$

The proof is complete.

Remark 2.3 If we do not define the topological degree, we may use $C(\bar{\Omega})$ and obtain similar results.

Remark 2.4 The difference between our Theorem 2.1 and Theorem 1 in [26] is that $F(x, u)$ can change sign and we get the existence of classical solutions to (2.1).

Remark 2.5 In the particular case $N=1$, we can also allow $p=1$, and it is classical that, in this case, $A$ also is completely continuous.

Remark 2.6 The difference between Definition 2.1 and the definitions in [26] is that we define a special function $\chi$ and the classical sub-supersolutions and that in [26] the subsupersolutions are in the sense of distribution.

In the following sections, we suppose that $a(t):[0,+\infty)$ is continuous and increasing on $[0,+\infty)$ for convenience.

\section{The existence of positive solutions with concave and convex nonlinearities}

In this section, we consider the problem

$$
\left\{\begin{array}{l}
-\Delta u=\frac{1}{a\left(\int_{\Omega}|u|^{\gamma} d x\right)}\left(\lambda u^{q}+u^{p}\right), \quad x \in \Omega \\
u(x)>0, \quad x \in \Omega \\
\left.u\right|_{\partial \Omega}=0
\end{array}\right.
$$

where $\gamma>0,1>q>0, p>0, \Omega=\left\{x \in R^{N}|| x \mid<1\right\}$.

In order to consider the existence of positive solutions for $(3.1)_{\lambda}$, we list some previous results. Let $\varphi_{1}$ be the eigenfunction corresponding to the principle eigenvalue of

$$
\left\{\begin{array}{l}
-\Delta u=\lambda u, \quad x \in \Omega, \\
\left.u\right|_{\partial \Omega}=0 .
\end{array}\right.
$$


It is found that $\lambda_{1}>0$ and $\varphi_{1}(x)>0$ for $x \in \Omega$; see [16]. Moreover, there exist $u, u^{*} \in C^{2}(\bar{\Omega})$ that satisfy

$$
\left\{\begin{array}{l}
-\Delta u=1, \quad x \in \Omega \\
u>0, \quad x \in \Omega \\
\left.u\right|_{\partial \Omega}=0
\end{array}\right.
$$

and

$$
\left\{\begin{array}{l}
-\Delta u=u^{q}, \quad x \in \Omega \\
u>0, \quad x \in \Omega \\
\left.u\right|_{\partial \Omega}=0
\end{array}\right.
$$

respectively. By [19] the following results are true:

$$
\frac{\varphi_{1}}{e} \in C(\bar{\Omega}), \quad \frac{\varphi_{1}}{u^{*}} \in C(\bar{\Omega}) .
$$

Suppose that $u_{\lambda}$ is a positive solution to $(3.1)_{\lambda}$. Let

$$
c=a\left(\int_{\Omega}\left|u_{\lambda}^{\gamma}\right| d x\right)^{-\frac{1}{p-1}} \text { and } v=c u_{\lambda} .
$$

Then $v$ satisfies

$$
\left\{\begin{array}{l}
-\Delta v=\lambda \frac{1}{\left(a\left(\int_{\Omega}|u(x)|^{\gamma} d x\right)\right)^{(p-q) /(p-1)}} v^{q}+v^{p}, \quad x \in \Omega, \\
\left.v\right|_{\partial \Omega}=0
\end{array}\right.
$$

and the transform (3.3) will be used later.

Let

$$
K=\left\{u \in C^{1}(\bar{\Omega}) \mid u(x) \geq 0, \forall x \in \bar{\Omega}\right\} .
$$

Obviously, $K$ is cone in $C^{1}(\bar{\Omega})$.

Using Theorem 2.1, we have following theorems.

Theorem 3.1 Assume that $\frac{2 N}{N-2}>p>1$ and $\lim _{t \rightarrow+\infty} \frac{t^{(p-1) / \gamma}}{a(t)}=+\infty$. Then there exist $\Gamma_{1} \geq$ $\Gamma_{2}>0$ such that

(1) (3.1) $)_{\lambda}$ has at least two positive solutions if $\lambda \in\left(0, \Gamma_{2}\right)$;

(2) $(3.1)_{\lambda}$ has at least one positive solution if $\lambda=\Gamma_{1}$ and $\lambda=\Gamma_{2}$;

(3) (3.1) has no positive solutions if $\lambda>\Gamma_{1}$.

Moreover,

$$
\left|u_{\lambda}\right|_{\infty} \leq C, \quad \forall \text { positive solutions } u_{\lambda} \text { to }(3.1)_{\lambda}, \lambda \in\left[0, \Gamma_{1}\right] .
$$

Theorem 3.2 Assume that $0<q<p<1$. Then $(3.1)_{\lambda}$ has at least one positive solution for $\lambda \geq 0$. 
Now we consider

$$
\left\{\begin{array}{l}
-\Delta u=\frac{1}{a_{0}}\left(\lambda u^{q}+u^{p}\right) \\
u(x)>0, \quad x \in \Omega \\
\left.u\right|_{\partial \Omega}=0
\end{array}\right.
$$

where $a_{0}=\inf _{t \in[0,+\infty)} a(t)$.

Lemma 3.1 (see [16]) Assume that $0<q<1, p>1$. Then there exist $\Gamma_{a_{0}}$ and $C_{a_{0}}>0$ such that

(1) $(3.5)_{\lambda}$ has at least two positive solutions if $\lambda \in\left(0, \Gamma_{a_{0}}\right)$;

(2) $(3.5)_{\lambda}$ has at least one positive solution if $\lambda=\Gamma_{a_{0}}$;

(3) $(3.5)_{\lambda}$ has no positive solutions if $\lambda>\Gamma_{a_{0}}$.

Moreover,

$$
\left|u_{\lambda}\right|_{\infty} \leq C_{a_{0}}, \quad \forall \text { positive solutions } u_{\lambda} \text { to }(3.5)_{\lambda}, \lambda \in\left[0, \Gamma_{a_{0}}\right]
$$

Lemma 3.2 (see [21]) Suppose that $f: \Omega \times R^{+} \rightarrow R$ is a continuous function such that $s^{-1} f(x, s)$ is strictly decreasing for $s>0$ at each $x \in \Omega$. Let $w, v \in C(\bar{\Omega}) \cap C^{2}(\Omega)$ satisfy:

(a) $\Delta w+f(x, w) \leq 0 \leq \Delta v+f(x, v)$ in $\Omega$;

(b) $w, v>0$ in $\Omega$, and $w \geq v$ on $\partial \Omega$;

(c) $\Delta v \in L^{1}(\Omega)$.

Then $w \geq v$ in $\bar{\Omega}$.

Proof of Theorem 3.1 (1) We show that for $\lambda \in\left(0, \Gamma_{a_{0}}\right),(3.1)_{\lambda}$ has at least one positive solution.

For $u \in P$, we define the operator

$$
\left(A_{\lambda} u\right)(x)=\frac{1}{a\left(\int_{\Omega}|u(x)|^{\gamma} d x\right)} \int_{\Omega} G(x, y)\left[\lambda u(y)^{q}+u(y)^{p}\right] d y, \quad x \in \bar{\Omega},
$$

where $G(x, y)$ is the Green function for $-\Delta u=h$.

For $\lambda \in\left(0, \Gamma_{a_{0}}\right)$, by [16] there exists a $u_{\lambda} \in C^{1}(\bar{\Omega})$ such that

$$
\left\{\begin{array}{l}
-\Delta u_{\lambda}=\frac{1}{a_{0}}\left(\lambda u_{\lambda}^{q}+u_{\lambda}^{p}\right), \quad x \in \Omega, \\
u_{\lambda}(x)>0, \quad x \in \Omega, \\
\left.u\right|_{\partial \Omega}=0
\end{array}\right.
$$

with $\frac{\partial u_{\lambda}}{\partial n}<0, x \in \partial \Omega$. Let $\beta(x)=u_{\lambda}$ and $b_{0}=\sup _{t \in\left[0,|\beta|_{\infty}^{\gamma}|\Omega|\right]} a(t)$. Since $0<1<q$ and $\lambda>0$, we can choose $\varepsilon>0$ small enough such that

$$
\begin{aligned}
& \varepsilon \lambda_{1} \varphi_{1}(x)<\frac{1}{b_{0}}\left(\lambda\left(\varepsilon \varphi_{1}(x)\right)\right)^{q}, \quad x \in \Omega, \\
& \varepsilon \varphi_{1}(x)<\beta(x), \quad \forall x \in \Omega,
\end{aligned}
$$

and

$$
\frac{\partial \varepsilon \varphi_{1}(x)}{\partial n}>\frac{\partial \beta(x)}{\partial n}, \quad \forall x \in \partial \Omega
$$


Let $\alpha(x)=\varepsilon \varphi_{1}(x)$. Then

$$
\left\{\begin{aligned}
-\Delta\left(\varepsilon \varphi_{1}(x)\right) & =\varepsilon \lambda_{1} \varphi_{1}(x) \\
& <\frac{1}{b_{0}}\left(\lambda\left(\varepsilon \varphi_{1}(x)\right)^{q}+\left(\varepsilon \varphi_{1}(x)\right)^{p}\right), \quad x \in \Omega, \\
\left.\varepsilon \varphi_{1}(x)\right|_{\partial \Omega}= & 0 .
\end{aligned}\right.
$$

Therefore, by the strict monotonicity of $a$ we have

$$
\left\{\begin{aligned}
-\Delta \beta(x) & =\frac{1}{a_{0}}\left(\lambda \beta(x)^{q}+\beta(x)^{p}\right) \\
& \geq \frac{1}{a\left(\int_{\Omega} \chi(x, u(x))^{\gamma} d x\right)}\left(\lambda \beta(x)^{q}+\beta(x)^{p}\right), \quad x \in \Omega, u \in C^{1}(\bar{\Omega}) \cap C^{2}(\Omega), \\
\beta(x)>0, \quad x \in \Omega, & \\
\left.\beta(x)\right|_{\partial \Omega} & =0
\end{aligned}\right.
$$

and

$$
\left\{\begin{aligned}
-\Delta(\alpha(x)) & =\varepsilon \lambda_{1} \varphi_{1}(x) \\
& <\frac{1}{b_{0}}\left(\lambda\left(\varepsilon \varphi_{1}(x)\right)^{q}+\left(\varepsilon \varphi_{1}(x)\right)^{p}\right) \\
& \leq \frac{1}{a\left(\int_{\Omega} \chi(x, u(x))^{\gamma} d x\right)}\left(\lambda\left(\varepsilon \varphi_{1}(x)\right)^{q}+\left(\varepsilon \varphi_{1}(x)\right)^{p}\right), \quad x \in \Omega, u \in C^{1}(\bar{\Omega}) \cap C^{2}(\Omega), \\
\left.\alpha\right|_{\partial \Omega}=0, &
\end{aligned}\right.
$$

with

$$
\frac{\partial \alpha}{\partial n}>\frac{\partial \beta(x)}{\partial n}, \quad \forall x \in \partial \Omega,
$$

which implies that $\alpha$ and $\beta$ are the subsolution and supersolution of (1.2) with $\alpha \prec \beta$. Now Theorem 2.1 implies that (3.1) $)_{\lambda}$ has at least one positive solution $u_{\lambda}$ with $\alpha(x) \leq u_{\lambda}(x) \leq$ $\beta(x), x \in \bar{\Omega}$. Moreover, if $W=\left\{u \in K \subseteq C^{1}(\bar{\Omega}) \mid \alpha \prec u \prec \beta\right\}$ and $u \in[\alpha, \beta]$ is a solution to (1.2), then we have

$$
\left\{\begin{array}{l}
-\Delta(u(x)-\alpha(x))>0, \quad x \in \Omega, \\
\left.(u-\alpha)\right|_{\partial \Omega}=0,
\end{array}\right.
$$

which, together with the maximum principle, means that

$$
\frac{\partial u(x)}{\partial n}<\frac{\partial \alpha(x)}{\partial n}, \quad x \in \partial \Omega
$$

that is,

$$
\alpha \prec u \text {. }
$$

A similar argument shows that

$$
u \prec \beta .
$$

Now Theorem 2.1 guarantees that

$$
i\left(A_{\lambda}, W, K\right)=\operatorname{deg}\left(I-A_{\lambda}, W, \theta\right)=1 .
$$


Let

$$
\Gamma_{1}=\sup \left\{\lambda>0:(3.1)_{\lambda} \text { has at least one positive solution }\right\}
$$

Obviously, $\Gamma_{1}>0$.

(2) We show that $\Gamma_{1}<+\infty$.

Assume that $u_{\lambda}$ is a solution to $(3.1)_{\lambda}$. Let $c=a\left(\int_{\Omega}\left|u_{\lambda}\right|^{\gamma} d x\right)^{-\frac{1}{p-1}}$ and $v=c u_{\lambda}$. Then we get (3.4). By Lemma 3.1 there exist $C^{\prime}>0$ and $\Gamma^{\prime}>0$ such that equation

$$
\left\{\begin{array}{l}
-\Delta v=\lambda v^{q}+v^{p} \\
\left.v\right|_{\partial \Omega}=0
\end{array}\right.
$$

has at least one positive solution for all $0 \leq \lambda \leq \Gamma^{\prime}$ and

$$
\sup _{\lambda \in\left[0, \Gamma^{\prime}\right]}\left|v_{\lambda}\right|_{\infty} \leq C^{\prime}
$$

which, together with (3.4), implies that

$$
\begin{aligned}
& \lambda \frac{1}{\left(a\left(\int_{\Omega}\left|u_{\lambda}(x)\right|^{\gamma} d x\right)\right)^{(p-q) /(p-1)}} \leq \Gamma^{\prime}, \\
& |v|_{\infty}=\left|a\left(\int_{\Omega}\left|u_{\lambda}(x)\right|^{\gamma} d x\right)^{-\frac{1}{p-1}} u\right|_{\infty} \leq C^{\prime},
\end{aligned}
$$

and

$$
|u|_{\infty} \leq a\left(\int_{\Omega}\left|u_{\lambda}(x)\right|^{\gamma} d x\right)^{\frac{1}{p-1}} C^{\prime}
$$

Now we show that $\left\{\int_{\Omega}\left|u_{\lambda}(x)\right|^{\gamma} d x: \lambda \in\left(0, \Gamma_{1}\right)\right\}$ is bounded.

In fact, if $\left\{\int_{\Omega}\left|u_{\lambda}(x)\right|^{\gamma} d x: \lambda \in\left(0, \Gamma_{1}\right)\right\}$ is unbounded, then there exists a sequence $\left\{u_{\lambda_{n}}\right\}$ such that

$$
\lim _{n \rightarrow+\infty} \int_{\Omega}\left|u_{\lambda_{n}}(x)\right|^{\gamma} d x=+\infty .
$$

Now (3.7) means that

$$
0 \leq a\left(\int_{\Omega}\left|u_{\lambda_{n}}(x)\right|^{\gamma} d x\right)^{-\frac{1}{p-1}} u_{\lambda_{n}}(x) \leq C^{\prime}, \quad x \in \Omega .
$$

Then

$$
0 \leq a\left(\int_{\Omega}\left|u_{\lambda_{n}}(x)\right|^{\gamma} d x\right)^{-\frac{\gamma}{p-1}} u_{\lambda_{n}}^{\gamma}(x) \leq C^{\prime \gamma}, \quad x \in \Omega .
$$

Integration on $\Omega$ yields that

$$
0 \leq a\left(\int_{\Omega}\left|u_{\lambda_{n}}(x)\right|^{\gamma} d x\right)^{-\frac{\gamma}{p-1}} \int_{\Omega}\left|u_{\lambda_{n}}(x)\right|^{\gamma} d x \leq|\Omega| C^{\prime \gamma} .
$$


Let $s_{n}=\int_{\Omega}\left|u_{\lambda_{n}}(x)\right|^{\gamma} d x$. Then

$$
\left(\frac{s_{n}^{(p-1) / \gamma}}{a\left(s_{n}\right)}\right)^{\frac{\gamma}{p-1}} \leq|\Omega| C^{\prime \gamma}
$$

which contradicts to

$$
\lim _{t \rightarrow+\infty} \frac{t^{(p-1) / \gamma}}{a(t)}=+\infty
$$

Since $a(t)>0$ is continuous on $[0,+\infty)$ with $\inf _{t \geq 0} a(t)=a_{0}>0$, the boundedness of $\left\{\int_{\Omega}\left|u_{\lambda}(x)\right|^{\gamma} d x: \lambda \in\left(0, \Gamma_{1}\right)\right\}$ means that

$$
a\left(\int_{\Omega}\left|u_{\lambda}(x)\right|^{\gamma} d x\right) \text { is bounded, }
$$

which, together with (3.8), means that

$$
\lambda \leq \Gamma^{\prime} \sup _{\lambda \in \Gamma_{1}}\left(a\left(\int_{\Omega}\left|u_{\lambda}(x)\right|^{\gamma} d x\right)\right)^{(p-q) /(p-1)} .
$$

Hence,

$$
\Gamma_{1}<+\infty
$$

From (3.9) we have

$$
\left|u_{\lambda}\right|_{\infty} \leq a\left(\int_{\Omega}\left|u_{\lambda}(x)\right|^{\gamma} d x\right)^{\frac{1}{p-1}} C^{\prime} \stackrel{\text { def }}{=} C<+\infty
$$

(3) We show that there exists $u_{\Gamma_{1}}$ satisfying (3.1) $\Gamma_{\Gamma_{1}}$. By the definition of $\Gamma_{1}>0$ there exists a sequence $\lambda_{n} \rightarrow \Gamma_{1}$ and $u_{\lambda_{n}}$ is a positive solution of (3.1) $)_{\lambda_{n}}$. From (3.10), there exists $C_{1}>0$ such that

$$
\left|\Delta u_{\lambda_{n}}(x)\right| \leq \frac{1}{a_{0}}\left[\lambda u_{\lambda_{n}}(x)^{q}+u_{\lambda_{n}}(x)^{p}\right] \leq C_{1}, \quad \forall \lambda_{n} \in\left[0, \Gamma_{1}\right],
$$

which guarantees that $\left\{u_{\lambda_{n}}\right\}$ is relatively compact in $C^{1}(\bar{\Omega})$. Then there exists $u_{\Gamma_{1}} \in C^{1}(\bar{\Omega})$ such that

$$
\lim _{n_{i} \rightarrow+\infty} u_{\lambda_{n_{i}}}(x)=u_{\Gamma_{1}}(x) \quad \text { uniformly on } \bar{\Omega} \text {. }
$$

A standard bootstrap argument shows that $u_{\Gamma_{1}} \in C^{1}(\bar{\Omega}) \cap C^{2}(\Omega)$ is a nonnegative solution for $(3.1)_{\Gamma_{1}}$.

(4) We show that for $\lambda \in\left(0, \Gamma_{a_{0}}\right),(3.1)_{\lambda}$ has at least two positive solutions.

By (3.10) and the Green formula there exists $C_{2}>0$ such that

$$
\begin{aligned}
\left|\nabla u_{\lambda}(x)\right| & =\frac{1}{a\left(\int_{\Omega}\left|u_{\lambda}(x)\right|^{\gamma} d x\right)}\left|\int_{\Omega} G_{x}(x, y)\left[\lambda u_{\lambda}(y)^{q}+u_{\lambda}(y)^{p}\right] d y\right| \\
& \leq C_{2}, \quad \forall \lambda \in\left[0, \Gamma_{1}\right] .
\end{aligned}
$$


Let $R>\max \left\{C, C_{2}\right\}$ and $\bar{\lambda}>\Gamma$. Let $H(\tau, u)=u-(-\Delta)^{-1}\left((\tau \lambda+(1-\tau) \bar{\lambda}) u^{q}+u^{p}\right)$ and $B_{R}=$ $\{u \mid\|u\|<R\}$. If there exist $\tau_{0} \in[0,1]$ and $u_{0} \in K \cap \partial B_{R}$ such that

$$
H\left(\tau_{0}, u_{0}\right)=u_{0}-(-\Delta)^{-1}\left(\left(\tau_{0} \lambda+\left(1-\tau_{0}\right) \bar{\lambda}\right) u_{0}^{q}+u_{0}^{p}\right)=0
$$

then we have

$$
\left\{\begin{array}{l}
-\Delta u_{0}=\frac{1}{a\left(\int_{\Omega}\left|u_{0}(x)\right|^{\gamma} d x\right)}\left(\left(\tau_{0} \lambda+\left(1-\tau_{0}\right) \bar{\lambda}\right) u^{q}+u_{0}^{p}\right), \quad x \in \Omega \\
u_{0}(x)>0, \quad x \in \Omega \\
\left.u_{0}\right|_{\partial \Omega}=0
\end{array}\right.
$$

which, together with (3.11) and (3.12), means that $\|u\|=\max \left\{\left|u_{0}\right|_{\infty},\left|\nabla u_{0}\right|_{\infty}\right\}<R$. This contradicts to $u_{0} \in\left(\partial B_{R}\right) \cap K$. The homotopy of $H$,

$$
\operatorname{deg}\left(I-H(1, \cdot), B_{R} \cap K, \theta\right)=\operatorname{deg}\left(I-H(0, \cdot), B_{R} \cap K, \theta\right) .
$$

Next, we claim that

$$
\operatorname{deg}\left(I-H(0, \cdot), B_{R} \cap K, \theta\right)=0 .
$$

In fact, suppose that there exist $1 \geq \mu_{0} \geq 0$ and $u_{0} \in \partial B_{R} \cap K$ such that $H\left(0, u_{0}\right)=\mu_{0} u_{0}$. Obviously, $\mu_{0}>0$ and $u_{0}$ satisfy

$$
\left\{\begin{array}{l}
-\Delta u_{0}=\frac{1}{\mu_{0} a\left(\int_{\Omega}\left|u_{0}(x)\right|^{\gamma} d x\right)}\left(\bar{\lambda} u_{0}^{q}+u_{0}^{p}\right), \quad x \in \Omega \\
u_{0}(x)>0, \quad x \in \Omega \\
\left.u_{0}\right|_{\partial \Omega}=0
\end{array}\right.
$$

Let $v=\mu_{0}^{-\frac{1}{p-1}} u_{0}$. Then $v$ satisfies that

$$
\left\{\begin{array}{l}
-\Delta v=\frac{1}{a\left(\int_{\Omega}\left|u_{0}(x)\right|^{\gamma} d x\right)}\left(\frac{1}{\mu_{0}^{(p-q) /(p-1)}} \bar{\lambda} v^{q}+\nu^{p}\right), \quad x \in \Omega \\
v(x)>0, \quad x \in \Omega \\
\left.v\right|_{\partial \Omega}=0 .
\end{array}\right.
$$

Since

$$
\frac{1}{\mu_{0}^{(p-q) /(p-1)}} \bar{\lambda}>\bar{\lambda}
$$

this contradicts the definition of $\Gamma_{1}$, which means that

$$
H(0, u) \neq \mu u, \quad \forall \mu \in[0,1], u \in \partial B_{R} \cap K .
$$

Therefore, (3.13) is true, and so,

$$
\operatorname{deg}\left(I-A,\left(B_{R}-\bar{W}\right) \cap K, \theta\right)=\operatorname{deg}\left(I-A, B_{R}, \theta\right)-\operatorname{deg}(I-A, W, \theta)=-1 .
$$


Now for $\lambda \in\left(0, \Gamma_{a_{0}}\right)$, we consider

$$
\left\{\begin{array}{l}
-\Delta u=\lambda u^{q}, \quad x \in \Omega, \\
u(x)>0, \quad x \in \Omega, \\
\left.u\right|_{\partial \Omega}=0 .
\end{array}\right.
$$

From [16], (3.15) has one positive solution $v_{0}$. Let $r=\left\|v_{0}\right\|$. Let $0<r<\min \left\{a\left(C^{\gamma}|\Omega|\right)^{\frac{1}{q-1}}\left\|v_{0}\right\|\right.$, $\|e\|\}$. For $\tau \in[0,1]$, define

$$
H(\tau, u)=u-(-\Delta)^{-1}\left(\lambda u^{q}+u^{p}+\tau\right), \quad u \in C^{1}(\bar{\Omega}) \cap K .
$$

We claim that

$$
H(\tau, u) \neq \theta, \quad \tau \in[0,1], u \in K \cap \partial B(0, r) .
$$

In fact, suppose $\left(\tau_{0}, u_{0}\right) \in[0,1] \times K \cap \partial B(0, r)$. Then

$$
\left\{\begin{array}{l}
-\Delta u_{0}=\frac{1}{a\left(\int_{\Omega}\left|u_{0}\right|^{\gamma} d x\right)}\left(\lambda_{0} u_{0}^{q}+u_{0}^{p}+\tau_{0}\right), \quad x \in \Omega \\
\left.u_{0}\right|_{\partial \Omega}=0 .
\end{array}\right.
$$

Let $c=a\left(\int_{\Omega} u_{0}^{\gamma} d x\right)^{-\frac{1}{q-1}}$ and $v=c u_{0}$. Then

$$
\left\{\begin{array}{l}
-\Delta v=\lambda v^{q}+c^{q-p} \nu^{p}+c^{q} \tau_{0} \\
\left.v\right|_{\partial \Omega}=0
\end{array}\right.
$$

By Lemma 3.2 we have

$$
v=c u_{\lambda} \geq v_{0}, \quad \forall \lambda \geq 0, x \in \bar{\Omega},
$$

and so

$$
u_{0} \geq a\left(C^{\gamma}|\Omega|\right)^{\frac{1}{q-1}} v_{0}
$$

which contradicts to $\left\|u_{0}\right\|=r<a\left(C^{\gamma}|\Omega|\right)^{\frac{1}{q-1}}\left\|v_{0}\right\|$. From the homotopy of $H$ it follows that

$$
\operatorname{deg}\left(I-A, B_{r} \cap K, \theta\right)=\operatorname{deg}\left(H(0, \cdot), B_{r} \cap K, \theta\right)=\operatorname{deg}\left(H(1, \cdot), B_{r} \cap K, \theta\right)=0,
$$

which, together with (3.14), implies that

$$
\operatorname{deg}\left(I-A,\left(B_{R}-\overline{\left(W \cup B_{r}\right)}\right) \cap K, \theta\right)=-1
$$

Consequently, $A$ has another fixed point $u_{\lambda, 1} \in\left(B_{R}-\overline{\left(W \cup B_{r}\right)}\right) \cap K$, that is, $(3.1)_{\lambda}$ has another positive solution $u_{\lambda, 1}$ for all $\lambda \in\left(0, \Gamma_{a_{0}}\right)$.

Consequently,

$$
+\infty>\Gamma_{1} \geq \Gamma_{2} \geq \Gamma_{a_{0}}
$$

Similarly, $(3.1)_{\Gamma_{2}}$ also has at least one positive solution. The proof is complete. 
Proof of Theorem 3.2 For given $\lambda>0$, since $0<q<p<1$, there exists $K_{1}>0$ such that

$$
1>\frac{\lambda K_{1}^{q-1}|e|_{\infty}^{q}+K_{1}^{p-1}|e|_{\infty}^{p}}{a_{0}},
$$

that is,

$$
K_{1}>\frac{\lambda K_{1}^{q}|e|_{\infty}^{q}+K_{1}^{p}|e|_{\infty}^{p}}{a_{0}} .
$$

Let $\beta(x)=K_{1} e(x)$. Then

$$
\left\{\begin{aligned}
-\Delta(\beta(x)) & =-\Delta\left(K_{1} e(x)\right) \\
& =K_{1} \\
& >\frac{\lambda K_{1}^{q}|e|_{\infty}^{q}+K_{1}^{p}|e|_{\infty}}{a_{0}}, \quad x \in \Omega, \\
\beta(x)=0, & x \in \partial \Omega .
\end{aligned}\right.
$$

Let $b_{0}=a\left(\int_{\Omega} \beta^{\gamma}(x) d x\right)$. Choose $\varepsilon>0$ small enough such that

$$
\varepsilon \lambda_{1} \varphi_{1}(x)<\frac{1}{b_{0}}\left(\lambda\left(\varepsilon \varphi_{1}(x)\right)\right)^{q}, \quad x \in \Omega,
$$

and

$$
\varepsilon \varphi_{1} \leq K_{1} e(x), \quad \forall x \in \bar{\Omega}
$$

Let $\alpha(x)=\varepsilon \varphi_{1}(x)$. Now (3.17), (3.18), and (3.19) guarantee that

$$
\left\{\begin{aligned}
-\Delta(\beta(x))> & \frac{\lambda K_{1}^{q}|e|_{\infty}^{q}+K_{1}^{p}|e|_{\infty}}{a_{0}} \\
& \geq \frac{\lambda(\beta(x))^{q}+(\beta(x))^{q}}{a\left(\int_{\Omega} \chi(x, u(x))^{\gamma} d x\right)}, \quad x \in \Omega, u \in C^{1}(\bar{\Omega}) \cap C^{2}(\Omega), \\
\beta(x)=0, & x \in \partial \Omega,
\end{aligned}\right.
$$

and

$$
\left\{\begin{aligned}
-\Delta(\alpha(x)) & =\varepsilon \lambda_{1} \varphi_{1}(x) \\
& <\frac{1}{b_{0}}\left(\lambda\left(\varepsilon \varphi_{1}(x)\right)^{q}+\left(\varepsilon \varphi_{1}(x)\right)^{p}\right) \\
& \leq \frac{1}{a\left(\int_{\Omega} \chi(x, u(x))^{\gamma} d x\right)}\left(\lambda(\alpha(x))^{q}+(\alpha(x))^{p}\right), \quad x \in \Omega, u \in C^{1}(\bar{\Omega}) \cap C^{2}(\Omega), \\
\left.\alpha(x)\right|_{\partial \Omega}= & 0,
\end{aligned}\right.
$$

which guarantees that $\alpha$ and $\beta$ are the subsolution and supersolution to (3.1) $)_{\lambda}$. Now Theorem 2.1 implies that $(3.1)_{\lambda}$ has at least one positive solution for all $\lambda \geq 0$. The proof is complete.

4 The existence of positive solutions when the nonlinearity is linear at $u=0$

In this section, we consider the problem

$$
\left\{\begin{array}{l}
-\Delta u=\frac{1}{a\left(\int_{\Omega}|u|^{\gamma} d x\right)}(\lambda u+f(x, u)), \quad x \in \Omega \\
u(x)>0, \quad x \in \Omega \\
\left.u\right|_{\partial \Omega}=0
\end{array}\right.
$$


where $\gamma>0$, and $\Omega \subseteq R^{N}$ is a bounded smooth domain. Now we list following conditions for convenience:

$\left(\mathrm{H}_{1}\right) f(x, u)$ is continuous on $\bar{\Omega} \times(-\infty,+\infty)$, and

$$
\lim _{|u| \rightarrow 0+} \frac{f(x, u)}{u}=0 \quad \text { uniformly on } x \in \bar{\Omega} .
$$

For $u \in C(\bar{\Omega})$, we define the operator

$$
\begin{aligned}
F(\lambda, u(x)) & =\frac{1}{a\left(\int_{\Omega}|u(x)|^{\gamma} d x\right)} \int_{\Omega} G(x, y)[\lambda u(y)+f(y, u(y))] d y \\
& =\frac{\lambda}{a_{0}} \int_{\Omega} G(x, y) u(y) d y+\int_{\Omega} G(x, y)\left[\frac{\lambda u(y)+f(y, u(y))}{a\left(\int_{\Omega}|u(x)|^{\gamma} d x\right)}-\frac{\lambda}{a_{0}} u(y)\right] d y \\
& \stackrel{\text { def }}{=} \lambda(L u)(x)+H(\lambda, u(x)), \quad x \in \bar{\Omega},
\end{aligned}
$$

where $G(x, y)$ is the Green function for $-\Delta u=h$.

Of course, under these new notation, $(\lambda, u)$ solves $(4.1)_{\lambda}$ if and only if

$$
u=F(\lambda ; u):=\lambda L u+H(\lambda, u) .
$$

Suppose that $\left(\mathrm{H}_{1}\right)$ holds. It is easy to see that $L: C(\bar{\Omega}) \rightarrow C(\bar{\Omega})$ is a compact and continuous linear operator and $H(\lambda, \cdot): C(\bar{\Omega}) \rightarrow C(\bar{\Omega})$ is a compact and continuous nonlinear operator. Moreover, (4.2) guarantees that

$$
\begin{aligned}
|H(\lambda, u(x))| & =\left|\int_{\Omega} G(x, y)\left[\frac{\lambda u(y)+f(y, u(y))}{a\left(\int_{\Omega}|u(x)|^{\gamma} d x\right)}-\frac{\lambda}{a_{0}} u(y)\right] d y\right| \\
& =\left|\int_{\Omega} G(x, y)\left[\frac{\lambda u(y)+f(y, u(y))}{a_{0}+o(1)}-\frac{\lambda}{a_{0}} u(y)\right] d y\right| \\
& =\left|\int_{\Omega} G(x, y)\left[\frac{\lambda u(y)+f(y, u(y))}{a_{0}} \frac{1}{1+o(1)}-\frac{\lambda}{a_{0}} u(y)\right] d y\right| \\
& =\left|\int_{\Omega} G(x, y)\left[\frac{\lambda u(y)+f(y, u(y))}{a_{0}}[1+o(1)]-\frac{\lambda}{a_{0}} u(y)\right] d y\right| \\
& =\left|\int_{\Omega} G(x, y) \frac{f(y, u(y))}{a_{0}}[1+o(1)] d y\right| \\
& =o(\|u\|), \quad \text { as }\|u\| \rightarrow 0 .
\end{aligned}
$$

Now, we state the following result.

Lemma 4.1 (see [31]) Let E be a Banach space. Suppose that $L$ is a compact linear operator and that $\lambda^{-1} \in \sigma(L)$ with odd multiplicity. If $H$ satisfies condition (4.4), then the set

$$
\Sigma=\overline{\{(\lambda ; u) \in \mathbb{R} \times E: u=\lambda L u+H(\lambda, u) ; u \neq 0\}}
$$

has a closed connected component $C=C_{\lambda}$ such that $(\lambda, 0) \in C$ and

(i) $C$ is unbounded in $\mathbb{R} \times E$, or

(ii) there exists $\hat{\lambda} \neq \lambda$ such that $(\hat{\lambda} ; 0) \in C$ and $\hat{\lambda}^{-1} \in \sigma(S)$. 
Suppose that $\lambda_{1}$ is the principle eigenvalue to the problem

$$
\left\{\begin{array}{l}
-\Delta u=\lambda \frac{1}{a_{0}} u, \quad x \in \Omega, \\
\left.u\right|_{\partial \Omega}=0 .
\end{array}\right.
$$

It is well known that the first eigenfunction $\phi_{1}$ associated to $\lambda_{1}$ can be chosen positive. Moreover, $\lambda_{1}$ is an eigenvalue with odd multiplicity.

By the global bifurcation theorem, $\left(\mathrm{H}_{1}\right)$ guarantees that there exists a closed connected component $C=C_{\lambda}$ of solutions for (4.1) $)_{\lambda}$ that satisfies (i) or (ii).

Lemma 4.2 There exists $\delta>0$ such that if $(\lambda, u) \in C$ with $\left|\lambda-\lambda_{1}\right|+|u|<\delta$ and $u \neq 0$, then $u$ has a defined sign, that is,

$$
u(x)>0, \quad x \in \Omega \quad \text { or } \quad u(x)<0, \quad x \in \Omega .
$$

Proof Take $\left\{u_{n}\right\}$ in $C(\bar{\Omega})$ and $\lambda_{n} \rightarrow \lambda_{1}$ such that

$$
u_{n} \neq 0, \quad\left\|u_{n}\right\| \rightarrow 0, \quad u_{n}=\lambda_{n} L u_{\lambda_{n}}+H\left(\lambda_{n}, u_{n}\right)
$$

Considering $w_{n}=u_{n} /\left\|u_{n}\right\|$, we get

$$
\left\{\begin{aligned}
-\Delta w_{n} & =\frac{\lambda_{n}}{a_{0}} w_{n}+\left[\frac{\left.\lambda_{n} w_{n}+\left.\frac{f\left(x, u_{n}\right)}{\|\left(\int_{\Omega}\left|u_{n}\right|\right.}\right|^{\gamma} d x\right)}{a_{0}}-\frac{\lambda_{n}}{a_{0}} w_{n}\right] \\
& =\frac{\lambda_{n}}{a_{0}} w_{n}+\left[\frac{\lambda_{n} w_{n}+o(1)}{a_{0}+o(1)}-\frac{\lambda_{n}}{a_{0}} w_{n}\right] \\
& =\frac{\lambda_{n}}{a_{0}} w_{n}+\left[\frac{o(1)}{a_{0}+o(1)}\right], \quad x \in \Omega, \\
w_{n}(x) & =0, \quad x \in \partial \Omega .
\end{aligned}\right.
$$

It is easy to check that

$$
\left\|w_{n}\right\|_{C^{1}(\bar{\Omega})} \leq \frac{1}{a_{0}}\left[\lambda_{n}+\left\|u_{n}\right\|_{C(\bar{\Omega})}+K\right],
$$

where $K$ is a positive constant.

Since that $\left\{u_{n}\right\}$ is bounded in $C(\bar{\Omega}),\left\{w_{n}\right\}$ is also bounded in $C^{1}(\bar{\Omega})$. By the Arzelà-Ascoli theorem, $\left\{w_{n}\right\}$ converges to some $w \in C^{1}(\bar{\Omega})$, uniformly in $\bar{\Omega}$, under a convenient subsequence. Of course, $\|w\|_{C(\bar{\Omega})}=1$, and thus $w \neq 0$ in $\Omega$.

Now, by (4.5) we know that $\left\{u_{n}\right\}$ is a Cauchy sequence in $C(\bar{\Omega})$ and

$$
w_{n}=\lambda_{n} L w_{n}+\frac{H\left(\lambda_{n}, u_{n}\right)}{\left\|u_{n}\right\|} .
$$

Letting $n \rightarrow+\infty$, we have

$$
w(x)=\frac{\lambda_{1}}{a_{0}} \int_{\Omega} G(x, y) w(y) d y,
$$

that is,

$$
\left\{\begin{array}{l}
-\Delta w=\frac{\lambda_{1}}{a_{0}} w, \quad x \in \Omega, \\
w(x)=0, \quad x \in \partial \Omega .
\end{array}\right.
$$


Since $w \neq 0$, by spectral theory we must have

$$
w(x)>0, \quad x \in \Omega \quad \text { or } \quad w(x)<0, \quad x \in \Omega .
$$

Without loss of generality, we can suppose that $w(x)>0$ for all $x \in \Omega$. Since $w$ is the $C^{1}(\bar{\Omega})$-limit of $\left\{w_{n}\right\}$, we must have $w_{n}(x)>0$ for all $x \in \Omega$ and $n$ large enough. Therefore, the sign of $u_{n}$ coincides with that of $w_{n}$ for $n$ large enough. The proof is complete.

Now we decompose $C$ into $C=C^{+} \cup C^{-}$, where

$$
C^{+}=\{(\lambda, u) \in C \mid u(x) \geq 0, \forall x \in \Omega\}
$$

and

$$
C^{-}=\{(\lambda, u) \in C \mid u(x) \leq 0, \forall x \in \Omega\}
$$

A simple computation gives that $C^{+}=\left\{(\lambda, u) \in C \mid(\lambda,-u) \in C^{-}\right\}$and $C^{+}$is unbounded if and only if $C$ is also unbounded.

Theorem 4.1 If $\left(\mathrm{H}_{1}\right)$ holds, then there exists an unbounded closed connected component $C=C_{\lambda}$ of solutions for $(4.1)_{\lambda}$.

Proof In fact, suppose that $C$ is bounded, which implies that $C^{+}$is bounded and $C$ contains $(\hat{\lambda}, 0)$, where $\hat{\lambda} \neq \lambda_{1}, \hat{\lambda}^{-1} \in \sigma(L)$.

In this way, we can take $\left\{u_{n}\right\}$ in $C(\bar{\Omega})$ and $\lambda_{n} \rightarrow \hat{\lambda}$ such that

$$
\lambda_{n} \neq 0, \quad\left\|u_{n}\right\| \rightarrow 0 \quad \text { and } \quad u_{n}=F\left(\lambda_{n}, u_{n}\right), \quad\left(\lambda_{n}, u_{n}\right) \in C^{+} .
$$

Considering $w_{n}=u_{n} /\left\|u_{n}\right\|_{C(\bar{\Omega})}$, we know that it satisfies problem (4.6). Moreover, as in the proof of Lemma 4.2, under an adequate subsequence, $\left\{w_{n}\right\}$ converges to $w$ in $C^{1}(\bar{\Omega})$, which is a nonzero solution of the eigenvalue problem

$$
\left\{\begin{array}{l}
-\Delta w=\hat{\lambda} \frac{1}{a_{0}} w, \quad x \in \Omega, \\
w=0 \text { on } \partial \Omega,
\end{array}\right.
$$

that is, $w$ is an eigenfunction related to $\hat{\lambda}$. Since $\hat{\lambda} \neq \lambda_{1}, w$ must change sign. Then, for $n$ large, each $w_{n}$ must change sign, and the same should hold for $u_{n}=w_{n}\left\|u_{n}\right\|_{C(\bar{\Omega})}$, which contradicts to $\left(\lambda_{n}, u_{n}\right) \in C^{+}$. The proof is complete.

Now we consider the following special problem:

$$
\left\{\begin{array}{l}
-\Delta u=\frac{1}{c_{1}+c_{2}\left(\int_{\Omega}|u|^{\gamma} d x\right)^{\alpha}}\left(\lambda u-|u|^{p-1} u\right), \quad x \in \Omega \\
u(x)>0, \quad x \in \Omega \\
\left.u\right|_{\partial \Omega}=0
\end{array}\right.
$$

where $\gamma>0, \Omega$ is a bounded smooth domain, and $p>1$.

By Theorem 4.1 the connected component $C^{+}$of $(4.7)_{\lambda}$ is unbounded. Now we have following theorem for $(4.7)_{\lambda}$. 
Theorem 4.2 Suppose that $p>\max \{\alpha \gamma+1, \gamma-1\}$. Then at least one positive solution of $(4.7)_{\lambda}$ exists if and only if $\lambda>\lambda_{1}$.

Proof First, we will show that for any $\Lambda>0$, there exists $r>0$ such that

$$
\|u\|_{H_{0}^{1}(\Omega)} \leq r, \quad \forall(\lambda, u) \in C^{+} \text {and } \lambda \leq \Lambda
$$

From now on, we denote by $\|\cdot\|$ the usual norm in $H_{0}^{1}(\Omega)$, that is,

$$
\|u\|=\|u\|_{H_{0}^{1}(\Omega)} \leq r
$$

Indeed, suppose that (4.8) is false. Then, there are $\left\{u_{n}\right\} \in H_{0}^{1}(\Omega)$ such that

$$
\left\|u_{n}\right\| \rightarrow+\infty \quad \text { and } \quad u_{n}=F\left(\lambda_{n}, u_{n}\right), \quad \lambda_{n} \leq \Lambda .
$$

Considering $w_{n}=u_{n} /\left\|u_{n}\right\|$, it follows from (4.3) that

$$
\begin{gathered}
\int_{\Omega} \nabla w_{n} \cdot \nabla v d x+\frac{1}{\left\|u_{n}\right\|} \frac{1}{a\left(\int_{\Omega} u_{n}(x)^{\gamma} d x\right)} \int_{\Omega} u_{n}^{p} v d x \\
=\frac{\lambda_{n}}{a\left(\int_{\Omega} u_{n}(x)^{\gamma} d x\right)} \int_{\Omega} w_{n} v d x, \quad \forall v \in H_{0}^{1}(\Omega) .
\end{gathered}
$$

Since that $\left\{w_{n}\right\}$ is bounded in $H_{0}^{1}(\Omega)$, without loss of generality, we can suppose that there is $w \in H_{0}^{1}(\Omega)$ satisfying

$$
w_{n} \rightarrow w \quad \text { in } H_{0}^{1}(\Omega), \quad w_{n} \rightarrow w \quad \text { in } L^{2}(\Omega),
$$

and

$$
w_{n}(x) \rightarrow w(x), \quad \text { a.e. in } \Omega .
$$

Taking $v=u_{n} /\left\|u_{n}\right\|^{p-\alpha \gamma}$ as a test function, (4.9) is

$$
\begin{gathered}
\frac{1}{\left\|u_{n}\right\|^{p-\alpha \gamma-1}}+\frac{\int_{\Omega} w_{n}^{p+1} d x}{c_{1}\left\|u_{n}\right\|^{-\alpha \gamma}+c_{2}\left(\int_{\Omega} w_{n}(x)^{\gamma} d x\right)^{\alpha}} \\
=\frac{\int_{\Omega} w_{n}^{2} d x}{\left\|u_{n}\right\|^{p-1-\alpha \gamma}\left[c_{1}+c_{2}\left(\int_{\Omega} u_{n}(x)^{\gamma} d x\right)^{\alpha}\right]} .
\end{gathered}
$$

Since $p>\alpha \gamma+1$, letting $n \rightarrow+\infty$, we derive

$$
\lim _{n \rightarrow+\infty} \frac{\int_{\Omega} w_{n}^{p+1} d x}{c_{1}\left\|u_{n}\right\|^{-\alpha \gamma}+c_{2}\left(\int_{\Omega} w_{n}(x)^{\gamma} d x\right)^{\alpha}}=0 .
$$

Since

$$
\frac{\int_{\Omega} w_{n}^{p+1} d x}{c_{1}\left\|u_{n}\right\|^{-\alpha \gamma}+c_{2}\left(\int_{\Omega} w_{n}(x)^{\gamma} d x\right)^{\alpha}} \geq \frac{\int_{\Omega} w_{n}^{p+1} d x}{c_{1}\left\|u_{n}\right\|^{-\alpha \gamma}+c_{2}\left(\int_{\Omega} w_{n}(x)^{p+1} d x\right)^{\frac{\alpha \gamma}{p+1}}|\Omega|^{\frac{\alpha(p+1-\gamma)}{p+1}}},
$$


we have

$$
\lim _{n \rightarrow+\infty} \frac{\int_{\Omega} w_{n}^{p+1} d x}{c_{1}\left\|u_{n}\right\|^{-\alpha \gamma}+c_{2}\left(\int_{\Omega} w_{n}(x)^{p+1} d x\right)^{\frac{\alpha \gamma}{p+1}}|\Omega|^{\frac{\alpha(p+1-\gamma)}{p+1}}}=0,
$$

which implies that

$$
\lim _{n \rightarrow+\infty} \int_{\Omega} w_{n}^{p+1} d x=0
$$

By the Fatou lemma

$$
\int_{\Omega} w(x)^{p+1} d x \leq \lim _{n \rightarrow+\infty} \int_{\Omega} w_{n}(x)^{p+1} d x=0 .
$$

Therefore, we should have $w=0$. Thereby, $\left\{w_{n}\right\}$ converges to 0 in $L^{2}(\Omega)$. Taking $v=w_{n}$ as a test function, we see that

$$
\begin{aligned}
& \int_{\Omega}\left|\nabla w_{n}\right|^{2}+\frac{1}{\left\|u_{n}\right\|\left[c_{1}+c_{2}\left(\int_{\Omega} u_{n}(x)^{\gamma} d x\right)^{\alpha}\right]} \int_{\Omega} u_{n}(x)^{p} w_{n}(x) d x \\
& \quad=\lambda_{n} \frac{1}{c_{1}+c_{2}\left(\int_{\Omega} u_{n}(x)^{\gamma} d x\right)^{\alpha}} \int_{\Omega} w_{n}(x)^{2} d x .
\end{aligned}
$$

Since $\left\{\lambda_{n}\right\}$ is bounded from above by $\Lambda$ and $\frac{1}{c_{1}+c_{2}\left(\int_{\Omega} u_{n}(x)^{\gamma} d x\right)^{\alpha}} \int_{\Omega} u_{n}(x)^{p} w_{n}(x) d x \geq 0$, we have

$$
\int_{\Omega}\left|\nabla w_{n}\right|^{2} \leq \frac{\Lambda}{c_{1}} \int_{\Omega} w_{n}(x)^{2} d x
$$

Taking the limit, we have that $\left\|w_{n}\right\| \rightarrow 0$, which contradicts to $\left\|w_{n}\right\|=1$ for all $n$. Then (4.8) is true, which, together with the boundedness of $\Omega$, implies that

$$
\|u\|_{C(\bar{\Omega})} \leq r, \quad \forall(\lambda, u) \in C^{+} \text {and } \lambda \leq \Lambda .
$$

Next, we will show the nonexistence of solution for $\lambda \leq \lambda_{1}$, proving that $C^{+}$does not intersect $\left[0, \lambda_{1}\right] \times H_{0}^{1}(\Omega)$. Indeed, suppose that

$$
(\lambda, u) \in\left[0, \lambda_{1}\right] \times H_{0}^{1}(\Omega), \quad(\lambda, u) \in C^{+} .
$$

Using $v=\phi_{1}$ as a test function in (4.6), we get

$$
\begin{aligned}
\frac{\lambda_{1}}{c_{1}+c_{2}\left(\int_{\Omega} u_{n}(x)^{\gamma} d x\right)^{\alpha}} \int_{\Omega} u \phi_{1} d x & >\frac{\lambda \int_{\Omega} u \phi_{1} d x-\int_{\Omega} u^{p} \phi_{1} d x}{c_{1}+c_{2}\left(\int_{\Omega} u_{n}(x)^{\gamma} d x\right)^{\alpha}} \\
& =\int_{\Omega} \nabla u \nabla \Phi_{1} d x=\frac{\lambda_{1}}{c_{1}} \int_{\Omega} u \phi_{1} d x
\end{aligned}
$$

This is a contradiction.

Consequently, problem $(4.7)_{\lambda}$ has at least one positive solution if and only if $\lambda>\lambda_{1}$. The proof is complete. 


\section{The positive solutions for singular nonlocal elliptic problems}

In this section, we consider the singular elliptic equation

$$
\left\{\begin{array}{l}
-a\left(\int_{\Omega}|u(x)|^{\gamma} d x\right) \Delta u(x)+K(x) u^{-\mu}=\lambda u^{q}, \quad x \in \Omega, \\
u(x)>0, \quad x \in \Omega \\
\left.u\right|_{\partial \Omega}=0
\end{array}\right.
$$

where $\gamma>0,1>q>0, \Omega$ is a bounded domain in $R^{N}, N \geq 2$, with $C^{2, \beta}$ boundary $\partial \Omega$, $\beta \in(0,1), K \in C^{2, \beta}(\bar{\Omega})$, and $0<q<1, \mu \in(0,1)$.

Now we list some previous results for the following equation:

$$
\left\{\begin{array}{l}
-\Delta u(x)+K(x) u^{-\mu}=\lambda u^{q}, \quad x \in \Omega \\
u(x)>0, \quad x \in \Omega \\
\left.u\right|_{\partial \Omega}=0
\end{array}\right.
$$

where $K \in C(\bar{\Omega})$ and $0<q<1, \mu \in(0,1)$. Define

$$
E=\left\{u \in C^{2, \beta}(\Omega) \cap C(\bar{\Omega}): u^{-\mu} \in L(\Omega)\right\} .
$$

Theorem 5.1 (see [21]) Let $K(x)<0, x \in \bar{\Omega}$. Then

(i) (5.2) $\lambda$ has a unique solution $u_{\lambda} \in E$ for any $\lambda \in R$;

(ii) $u_{\lambda}$ is increasing with respect to $\lambda$;

(iii) $c_{1} d(x) \leq u_{\lambda}(x) \leq c_{2} d(x)$ for any $x \in \Omega$ and some $c_{1}$ and $c_{2}>0$ independent of $x$;

(iv) $u_{\lambda} \in C^{1,1-\mu}(\Omega)$.

Theorem 5.2 (see [21]) Let $\min _{x \in \bar{\Omega}} K(x)>0$. Then

(i) there exists $\lambda^{*}>0$ such that $(5.2)_{\lambda}$ has at least one positive solution $u_{\lambda} \in E$ for any $\lambda>\lambda^{*}$;

(ii) $c_{1} d(x) \leq u_{\lambda}(x) \leq c_{2} d(x)$ for any $x \in \Omega$ and some $c_{1}$ and $c_{2}>0$ independent of $x$;

(iii) $u_{\lambda} \in C^{1,1-\mu}(\Omega)$.

Using Theorems 5.1 and 5.2, by Theorem 2.1 we have the following results for $(5.1)_{\lambda}$.

Theorem 5.3 Let $K(x)<0$ for all $x \in \bar{\Omega}$. Then

(i) (1.1) has at least one solution $u_{\lambda} \in E$ for any $\lambda \geq 0$;

(ii) $c_{1} d(x) \leq u_{\lambda}(x) \leq c_{2} d(x)$ for any $x \in \Omega$ and some $c_{1}$ and $c_{2}>0$ independent of $x$, and $u_{\lambda} \in C^{1,1-\mu}(\Omega)$.

Proof (1) For $\lambda \geq 0$, we consider the problem

$$
\left\{\begin{array}{l}
-a\left(\int_{\Omega}|u(x)|^{\gamma} d x\right) \Delta u(x)+K(x)\left(u(x)+\frac{1}{n}\right)^{-\mu}=\lambda u^{q}, \quad x \in \Omega, \\
u(x)>0, \quad x \in \Omega, \\
\left.u\right|_{\partial \Omega}=0
\end{array}\right.
$$

where $n \in\{1,2, \ldots\}$.

Since $\mu \in(0,1)$ and $0<q<1$, there exists $K_{1}>0$ such that

$$
1>\frac{\max _{x \in \bar{\Omega}}|K(x)| K_{1}^{-\alpha-1}+\lambda K_{1}^{q-1}|e+1|_{\infty}^{q}}{a_{0}},
$$


that is,

$$
K_{1}>\frac{\max _{x \in \bar{\Omega}}|K(x)| K_{1}^{-\mu}+\lambda K_{1}^{q}|e+1|_{\infty}^{q}}{a_{0}} .
$$

Let $\beta(x)=K_{1}(e(x)+1)$. Then

$$
\left\{\begin{aligned}
-\Delta(\beta(x)) & =-K_{1} \Delta(e(x)+1) \\
& =K_{1} \\
& >\frac{\max _{x \in \bar{\Omega}}|K(x)| K_{1}^{-\mu}+\lambda K_{1}^{q}|e+1|_{\infty}^{q}}{a_{0}} \\
& \geq \frac{-K(x)(\beta(x))^{-\mu}+\lambda(\beta(x))^{q}}{a_{0}}, \quad x \in \Omega, \\
\beta(x)=1, & x \in \partial \Omega .
\end{aligned}\right.
$$

Let $b_{0}=a\left(\int_{\Omega} \beta(x)^{\gamma} d x\right)$. Since $0<q<1$, there exists $\frac{1}{n}>\varepsilon_{n}>0$ small enough such that

$$
\varepsilon_{n} \lambda_{1} \varphi_{1}(x)<\frac{1}{b_{0}}\left(\min _{x \in \bar{\Omega}}(-K(x))\left(\varepsilon_{n} \varphi_{1}(x)+\frac{1}{n}\right)^{-\mu}+\lambda\left(\varepsilon_{n}\left(\varphi_{1}(x)\right)\right)^{q}\right), \quad x \in \Omega,
$$

and

$$
\varepsilon_{n} \varphi_{1}(x)<K_{1}(e(x)+1), \quad \forall x \in \bar{\Omega} .
$$

Let $\alpha_{n}(x)=\varepsilon_{n} \varphi_{1}(x)$. Then, for $u \in C^{1}(\bar{\Omega}) \cap C^{2}(\Omega)$, (5.4), (5.5), and (5.6) imply that

$$
\left\{\begin{aligned}
-\Delta(\beta(x)) & >\frac{\left.-K(x)(\beta(x))^{-\mu}+\lambda \beta(x)\right)^{q}}{a_{0}} \\
& \geq \frac{-K(x)\left(\left(\beta(x)+\frac{1}{n}\right)^{q}\right)^{-\mu}+\lambda \beta(x)^{q}}{a\left(\int_{\Omega} \chi(x, u(x))^{\gamma} d x\right)}, \quad x \in \Omega \\
\beta(x)=1 & >0, \quad x \in \partial \Omega
\end{aligned}\right.
$$

and

$$
\left\{\begin{aligned}
-\Delta\left(\alpha_{n}(x)\right) & =\varepsilon \lambda_{1} \varphi_{1}(x) \\
& <\frac{1}{b_{0}}\left(\min _{x \in \bar{\Omega}}(-K(x))\left(\varepsilon_{n} \varphi_{1}(x)+\frac{1}{n}\right)^{-\mu}+\left(\varepsilon_{n} \varphi_{1}(x)\right)^{q}\right) \\
& \leq \frac{1}{a\left(\int_{\Omega} x(x, u(x))^{\gamma} d x\right)}\left(-K(x)\left(\alpha_{n}(x)+\frac{1}{n}\right)^{-\mu}+\lambda\left(\alpha_{n}(x)\right)^{q}\right), \quad x \in \Omega, \\
\left.\alpha_{n}\right|_{\partial \Omega}=0, &
\end{aligned}\right.
$$

which means that $\alpha_{n}(x)$ and $\beta(x)$ are the subsolution and supersolution to (5.3) ${ }_{n}$. Now Theorem 2.1 implies that $(5.3)_{n}$ has at least one positive solution $u_{n}$.

Now we consider set $\left\{u_{n}\right\}$. Since $\left|u_{n}\right|_{\infty} \leq K_{1}|e+1|_{\infty}$, it follows that

$$
\lambda\left(u_{n}(x)+\frac{1}{n}\right)^{q}-K(x)\left(u_{n}(x)+\frac{1}{n}\right)^{-\mu} \geq \min _{x \in \bar{\Omega}}|K(x)|\left(K_{1}\left(|e+1|_{\infty}\right)+\frac{1}{n}\right)^{-\mu},
$$

and so

$$
\left\{\begin{aligned}
-\Delta\left(u_{n}(x)\right) & =\frac{1}{a\left(\int_{\Omega} u_{n}^{\gamma}(x) d x\right)}\left(\lambda u_{n}(x)^{q}-K(x)\left(u_{n}(x)+\frac{1}{n}\right)^{-\mu}\right) \\
& \geq \frac{1}{b_{0}} \min _{x \in \bar{\Omega}}|K(x)|\left(K_{1}\left(|e+1|_{\infty}\right)+\frac{1}{n}\right)^{-\mu}, \quad x \in \Omega, \\
\left.u_{n}\right|_{\partial \Omega}=0 . &
\end{aligned}\right.
$$


Lemma 3.2, together with (3.3), implies there exists $c_{0}>0$ such that

$$
u_{n}(x) \geq \frac{1}{b_{0}} \min _{x \in \bar{\Omega}}|K(x)|\left(K_{1}\left(|e+1|_{\infty}+1\right)\right)^{-\mu} e(x) \geq c_{0} \varphi_{1}(x), \quad x \in \bar{\Omega},
$$

where $b_{0}=a\left(\int_{\Omega} \beta^{\gamma}(x) d x\right)$. Then

$$
\left|\nabla u_{n}(x)\right| \leq \frac{1}{a_{0}} \int_{\Omega}\left|G_{x}(x, y)\right|\left(\max _{x \in \bar{\Omega}}|K(x)|\left(c_{0} \varphi_{1}(y)\right)^{-\mu}+\lambda\left(K_{1}|e+1|_{\infty}\right)^{q}\right) d y
$$

and

$$
\begin{aligned}
& \left|\nabla u_{n}\left(x_{1}\right)-\nabla u_{n}\left(x_{2}\right)\right| \\
& \quad \leq \frac{1}{a_{0}} \int_{\Omega}\left|G_{x}\left(x_{1}, y\right)-G_{x}\left(x_{2}, y\right)\right|\left(\max _{x \in \bar{\Omega}}|K(x)|\left(c_{0} \varphi_{1}(y)\right)^{-\mu}+\lambda\left(K_{1}|e+1|_{\infty}\right)^{q}\right) d y .
\end{aligned}
$$

The same technique as in [32], Theorem 1.1, yields

$$
\left|\nabla u_{n}\left(x_{1}\right)-\nabla u_{n}\left(x_{2}\right)\right| \leq C\left|x_{1}-x_{2}\right|^{1-\mu}
$$

Therefore, $u_{n} \in C^{1,1-\mu}(\bar{\Omega})$. The sequence $\left\{u_{n}\right\}$ has a subsequence $\left\{u_{n_{i}}\right\}$ such that

$$
\lim _{n_{i} \rightarrow+\infty} u_{n_{i}}=u_{\lambda} \quad \text { uniformly on } \bar{\Omega} \text {. }
$$

Now a straightforward calculation yields

$$
\left\{\begin{array}{l}
-\Delta\left(u_{\lambda}(x)\right)=\frac{1}{a\left(\int_{\Omega} u_{\lambda}^{\gamma}(x) d x\right)}\left(\lambda u_{\lambda}(x)^{q}-K(x) u(x)^{-\mu}\right), \quad x \in \Omega \\
\left.u_{\lambda}\right|_{\partial \Omega}=0 .
\end{array}\right.
$$

(2) Suppose that $u_{\lambda}$ satisfies (5.1) $)_{\lambda}$. Let $c_{0}=a\left(\int_{\Omega} u_{\lambda}^{\gamma}(x) d x\right)>0$ and $v(x)=a\left(\int_{\Omega} u_{\lambda}^{\gamma}(x) d x\right) \times$ $u_{\lambda}(x), x \in \bar{\Omega}$. Then $v(x)$ satisfies

$$
\left\{\begin{array}{l}
-\Delta v(x)+c_{0}^{\mu} K(x) u^{-\mu}=\frac{1}{c_{0}} \lambda u^{q}, \quad x \in \Omega, \\
v(x)>0, \quad x \in \Omega \\
\left.v\right|_{\partial \Omega}=0 .
\end{array}\right.
$$

Now Theorem 5.1 implies that there exist $c_{1}$ and $c_{2}>0$ independent of $x$ such that

$$
c_{1} d(x) \leq v_{\lambda}(x) \leq c_{2} d(x) \quad \text { for any } x \in \Omega
$$

and so

$$
\frac{1}{a\left(\int_{\Omega} u_{\lambda}^{\gamma}(x) d x\right)} c_{1} d(x) \leq u_{\lambda}(x) \leq \frac{1}{a\left(\int_{\Omega} u_{\lambda}^{\gamma}(x) d x\right)} c_{2} d(x) \quad \text { for any } x \in \Omega
$$

Moreover, $v \in C^{1,1-\mu}(\Omega)$ implies that $u \in C^{1,1-\mu}(\Omega)$. The proof is complete. 
Theorem 5.4 Let $\min _{x \in \bar{\Omega}} K(x)>0$. Suppose that there exists $\rho>\frac{1}{1-q}$ such that

$$
\lim _{t \rightarrow+\infty} a(t)=+\infty \quad \text { and } \quad \lim _{t \rightarrow+\infty} \frac{t^{1 /(\rho \gamma)}}{a(t)}=+\infty
$$

Then

(i) there exists $\lambda^{*}>0$,such that (1.1) has at least one positive solution $u_{\lambda} \in E$ for any $\lambda>\lambda^{*}$

(ii) $c_{1} d(x) \leq u_{\lambda}(x) \leq c_{2} d(x)$ for any $x \in \Omega$ and some $c_{1}$ and $c_{2}>0$ independent of $x$, and $u_{\lambda} \in C^{1,1-\mu}(\Omega)$.

Proof For $\varphi_{1}$, by the Hopf maximum principle, there exist $\delta_{0}>0$ and $\Sigma \subset \Omega$ such that

$$
\left|\nabla \varphi_{1}\right| \geq \delta_{1}, \quad x \in \Sigma, \quad\left|\varphi_{1}\right| \geq \delta_{0}, \quad x \in \Omega-\Sigma
$$

Then there exists $M_{1}>0$ such that

$$
\frac{M_{1} 2(1-\mu)\left|\nabla \varphi_{1}\right|}{(1+\mu)^{2} \varphi_{1}^{2 \mu /(1+\mu)}} \geq \frac{K^{*}}{M_{1}^{\mu} \varphi_{1}^{2 \mu /(1+\mu)}}, \quad x \in \Sigma
$$

and there exists $M_{2}>0$ such that

$$
\frac{K^{*}}{M_{2}^{\mu} \varphi_{1}^{2 \mu /(1+\mu)}} \leq \frac{\lambda_{1} M_{2}}{1+\mu} \varphi_{1}^{\frac{2}{1+\mu}}, \quad x \in \Omega-\Sigma,
$$

where $K^{*}=\max _{x \in \bar{\Omega}} K(x)$. By (5.7), choose $M>\max \left\{M_{1}, M_{2}\right\}$ large enough such that

$$
a\left(M^{\gamma} \int_{\Omega} \varphi_{1}(x)^{\frac{2 \gamma}{1+\mu}} d x\right)>1
$$

Combining (5.8) and (5.9), we have

$$
\begin{aligned}
-\Delta M \varphi_{1}^{\frac{2}{1+\mu}}+K(x)\left(M \varphi_{1}^{\frac{2}{1+\mu}}\right)^{-\mu} & =\left[\frac{2 M}{1+\mu} \lambda_{1} \varphi_{1}^{\frac{2}{1+\mu}}-\frac{2(1-\mu) M\left|\nabla \varphi_{1}\right|^{2}}{(1+\mu)^{2} \varphi_{1}^{2 \mu /(1+\mu)}}\right]+K(x) \frac{1}{M^{\mu} \varphi_{1}^{\frac{2 \mu}{1+\mu}}} \\
& \leq \frac{3 M}{1+\mu} \lambda_{1} \varphi_{1}^{\frac{2}{1+\mu}}, \quad x \in \Omega-\Sigma
\end{aligned}
$$

and so

$$
-\Delta M \varphi_{1}^{\frac{2}{1+\mu}} \leq \frac{3 M}{1+\mu} \lambda_{1} \varphi_{1}^{\frac{2}{1+\mu}}-K(x)\left(M \varphi_{1}^{\frac{2}{1+\mu}}\right)^{-\mu}, \quad x \in \Omega-\Sigma .
$$

Therefore, for $n>0$,

$$
\begin{aligned}
-\Delta\left(M \varphi_{1}^{\frac{2}{1+\mu}}\right) & \leq \frac{3 M}{1+\mu} \lambda_{1} \varphi_{1}^{\frac{2}{1+\mu}}-K(x)\left(M \varphi_{1}^{\frac{2}{1+\mu}}\right)^{-\mu} \\
& \leq \frac{3 M}{1+\mu} \lambda_{1}\left(\varphi_{1}^{\frac{2}{1+\mu}}\right)-K(x)\left(M\left(\varphi_{1}^{\frac{2}{1+\mu}}+\frac{1}{n}\right)\right)^{-\mu}, \quad x \in \Omega-\Sigma .
\end{aligned}
$$


Let $u_{1}^{*}(x)$ satisfy

$$
\left\{\begin{array}{l}
-\Delta u=u^{q}, \quad x \in \Omega \\
\left.u\right|_{\partial \Omega}=1
\end{array}\right.
$$

Now by (5.7) it follows that

$$
\lim _{t \rightarrow+\infty} \frac{t}{a\left(t^{\gamma \rho} \int_{\Omega} u_{1}^{*}(x)^{\gamma} d x\right)}=+\infty
$$

which implies that there exists $T_{0}>0$ such that, for all $t>T_{0}$,

$$
\frac{t}{a\left(t^{\gamma \rho} \int_{\Omega} u_{1}^{*}(x)^{\gamma} d x\right)}>\frac{3 M^{1-q}}{1+\mu} \lambda_{1} \varphi_{1}^{\frac{2-2 q}{1+\mu}}
$$

Let

$$
T_{1}>\max \left\{T_{0},\left(\sup _{x \in \bar{\Omega}} \frac{M\left(\varphi_{1}^{2 /(1+\mu)}(x)+1\right)}{u_{1}^{*}(x)}\right)^{1 / \rho}, a_{0}^{(-1) /(\rho(1-q)-1)}\right\} .
$$

For $\lambda>T_{1}$, let

$$
\underline{u}_{\lambda}(x)=M \varphi_{1}^{\frac{2}{1+\mu}}(x) \quad \text { and } \quad \bar{u}_{\lambda, 1}(x)=\lambda^{\rho} u_{1}^{*}(x), \quad x \in \bar{\Omega} .
$$

It is easy to see that

$$
\underline{u}_{\lambda}(x) \leq \bar{u}_{\lambda, 1}(x)=\lambda^{\rho} u_{1}^{*}(x), \quad x \in \bar{\Omega} .
$$

For $u \in C^{1}(\bar{\Omega})$, let

$$
\chi(x, u(x))=\underline{u}_{\lambda}(x)+\left(u(x)-\underline{u}_{\lambda}(x)\right)^{+}-\left(u(x)-\bar{u}_{\lambda, 1}(x)\right)^{+} .
$$

Then

$$
a_{0} \leq a\left(\int_{\Omega}|\chi(x, u(x))|^{\gamma} d x\right) \leq a\left(\lambda^{\gamma \rho} \int_{\Omega}\left(u_{1}^{*}(x)\right)^{\gamma} d x\right) .
$$

Let

$$
b_{0}=a\left(\lambda^{\gamma \rho} \int_{\Omega}\left(u_{1}^{*}(x)\right)^{\gamma} d x\right) .
$$

From (5.9) we know that $b_{0}>1$. By (5.11), (5.13), and (5.14), for $u \in C^{1}(\bar{\Omega}) \cap C^{2}(\Omega)$, we have

$$
\left\{\begin{aligned}
-\Delta\left(\bar{u}_{\lambda, 1}(x)\right) & =-\Delta\left(\lambda^{\rho} u_{1}^{*}(x)\right) \\
& =\lambda^{\rho-\rho q}\left(\lambda^{\rho} u_{1}^{*}(x)\right)^{q} \\
& =\lambda^{(1-q) \rho-1} \lambda\left(\bar{u}_{\lambda, 1}(x)\right)^{q} \\
& \geq \frac{1}{a_{0}} \lambda\left(\bar{u}_{\lambda, 1}(x)\right)^{q}-\frac{1}{a\left(\int_{\Omega}(\chi(x, u(x)))^{\gamma} d x\right)} K(x)\left(\bar{u}_{\lambda, 1}(x)+\frac{1}{n}\right)^{-\mu} \\
& \geq \frac{1}{a\left(\int_{\Omega}(\chi(x, u(x)))^{\gamma} d x\right)}\left[\lambda\left(\bar{u}_{\lambda, 1}(x)\right)^{q}-K(x)\left(\bar{u}_{\lambda, 1}(x)+\frac{1}{n}\right)^{-\mu}\right], \quad x \in \Omega, \\
\bar{u}_{\lambda, 1}(x)=\lambda^{\rho} & >0, \quad x \in \partial \Omega,
\end{aligned}\right.
$$


and

$$
\left\{\begin{aligned}
-\Delta\left(\underline{u}_{\lambda}(x)\right)= & -\Delta\left(M \varphi_{1}^{\frac{2}{1+\mu}}(x)\right) \\
\leq & {\left[\frac{3 M}{1+\mu} \lambda_{1} \varphi_{1}^{\frac{2}{1+\mu}}(x)-K(x)\left(M \varphi_{1}^{\frac{2}{1+\mu}}(x)\right)^{-\mu}\right] } \\
= & \frac{1}{b_{0}}\left[b_{0} \frac{3 M^{1-q}}{1+\mu} \lambda_{1} \varphi_{1}(x)^{\frac{2-2 q}{1+\mu}}\left(\underline{u}_{\lambda}(x)\right)^{q}\right. \\
& \left.-K(x) b_{0}\left(M \varphi_{1}^{\frac{2}{1+\mu}}(x)\right)^{-\mu}\right] \\
\leq & \frac{1}{a\left(\int_{\Omega}(\chi(x, u(x)))^{\gamma} d x\right)}\left[\lambda\left(\underline{u}_{\lambda}(x)\right)^{q}-K(x)\left(\underline{u}_{\lambda}(x)+\frac{1}{n}\right)^{-\mu}\right], \quad x \in \Omega, \\
\left.\underline{u}_{\lambda}(x)\right|_{\partial \Omega}= & 0 .
\end{aligned}\right.
$$

Hence, $\underline{u}_{\lambda}(x)$ and $\bar{u}_{\lambda}(x)$ are the subsolution and supersolution of (5.3) ${ }_{n}$. Now Theorem 2.1 implies that for $n \in\{1,2, \ldots\},(5.3)_{n}$ has at least one solution $u_{\lambda}$ with

$$
\underline{u}_{\lambda}(x) \leq u_{\lambda, n}(x) \leq \bar{u}_{\lambda, 1}(x), \quad x \in \bar{\Omega} .
$$

Now we consider set $\left\{u_{\lambda, n}\right\}$. From (5.14) we have

$$
\left|\nabla u_{\lambda, n}(x)\right| \leq \frac{1}{a_{0}} \int_{\Omega}\left|G_{x}(x, y)\right|\left(\max _{x \in \bar{\Omega}}|K(x)|\left(M \varphi_{1}(y)\right)^{-2 \mu /(1+\mu)}+\lambda\left(\lambda^{\rho}\left\|u_{1}^{*}\right\|\right)^{q}\right) d y
$$

and

$$
\begin{aligned}
& \left|\nabla u_{\lambda, n}\left(x_{1}\right)-\nabla u_{\lambda, n}\left(x_{2}\right)\right| \\
& \quad \leq \frac{1}{a_{0}} \int_{\Omega}\left|G_{x}\left(x_{1}, y\right)-G_{x}\left(x_{2}, y\right)\right|\left(\max _{x \in \bar{\Omega}}|K(x)|\left(M \varphi_{1}(y)\right)^{-2 \mu /(1+\mu)}+\lambda\left(\lambda^{\rho}\left\|u_{1}^{*}\right\|\right)^{q}\right) d y .
\end{aligned}
$$

The same technique as in [32], Theorem 1.1, yields

$$
\left|\nabla u_{\lambda, n}\left(x_{1}\right)-\nabla u_{\lambda, n}\left(x_{2}\right)\right| \leq C\left|x_{1}-x_{2}\right|^{1-2 \mu /(1+\mu)}
$$

Therefore, $u_{n} \in C^{1,1-2 \mu /(1+\mu)}(\bar{\Omega})$. The sequence $\left\{u_{n}\right\}$ has a subsequence $\left\{u_{n_{i}}\right\}$ such that

$$
\lim _{n_{i} \rightarrow+\infty} u_{n_{i}}=u_{\lambda} \quad \text { uniformly on } \bar{\Omega}
$$

Now a straightforward calculation yields

$$
\left\{\begin{array}{l}
-\Delta\left(u_{\lambda}(x)\right)=\frac{1}{a\left(\int_{\Omega} u_{\lambda}^{\gamma}(x) d x\right)}\left(\lambda u_{\lambda}(x)^{q}-K(x) u_{\lambda}(x)^{-\mu}\right), \quad x \in \Omega, \\
\left.u_{\lambda}\right|_{\partial \Omega}=0 .
\end{array}\right.
$$

(2) Suppose that $u_{\lambda}$ satisfies (5.1) $)_{\lambda}$. Let $c_{0}=a\left(\int_{\Omega} u_{\lambda}^{\gamma}(x) d x\right)>0$ and $v(x)=a\left(\int_{\Omega} u_{\lambda}^{\gamma}(x) d x\right) \times$ $u_{\lambda}(x), x \in \bar{\Omega}$. Then $v(x)$ satisfies

$$
\left\{\begin{array}{l}
-\Delta v(x)+c_{0}^{\mu} K(x) u^{-\mu}=\frac{1}{c_{0}^{q}} \lambda u^{q}, \quad x \in \Omega \\
v(x)>0, \quad x \in \Omega \\
\left.v\right|_{\partial \Omega}=0
\end{array}\right.
$$


Now Theorem 5.2 implies that there exist $c_{1}$ and $c_{2}>0$ independent of $x$ such that

$$
c_{1} d(x) \leq v_{\lambda}(x) \leq c_{2} d(x) \quad \text { for any } x \in \Omega
$$

and so

$$
\frac{1}{a\left(\int_{\Omega} u_{\lambda}^{\gamma}(x) d x\right)} c_{1} d(x) \leq u_{\lambda}(x) \leq \frac{1}{a\left(\int_{\Omega} u_{\lambda}^{\gamma}(x) d x\right)} c_{2} d(x) \quad \text { for any } x \in \Omega .
$$

Moreover, $v \in C^{1,1-\mu}(\Omega)$ implies that $u \in C^{1,1-\mu}(\Omega)$. The proof is complete.

\section{Positive solutions for (1.1) when $N=1$}

In this section, we consider the case $N=1$ :

$$
\left\{\begin{array}{l}
-u^{\prime \prime}=\frac{1}{a\left(\int_{\Omega}|u|^{p} d x\right)} f(x, u), \quad x \in(0,1), \\
u(0)=u(1)=0
\end{array}\right.
$$

and by using Theorem 2.1 we present sufficient and necessary conditions for the existence of positive solutions for (6.1).

Now we list some conditions for convenience:

$\left(\mathrm{H}_{1}\right) f:(0,1) \times(0,+\infty) \rightarrow(0,+\infty)$ is continuous, and there exist $\lambda, \mu, \delta(-\infty<\lambda<0<\mu<$ $1,0<\delta \leq 1)$ such that for all $x \in(0,1)$ and $v \in(0,+\infty)$, we have

$$
\begin{array}{ll}
c_{0}^{\mu} f(x, v) \leq f\left(x, c_{0} v\right) \leq c_{0}^{\lambda} f(x, v), & 0 \leq c_{0} \leq \delta \\
c_{0}^{\lambda} f(x, v) \leq f\left(x, c_{0} v\right) \leq c_{0}^{\mu} f(x, v), & c_{0} \geq 1 / \delta
\end{array}
$$

Now we state a result on the existence of positive solutions for the following problem from [33]

$$
\left\{\begin{array}{l}
-u^{\prime \prime}=f(x, u), \quad x \in(0,1), \\
u(0)=u(1)=0
\end{array}\right.
$$

Theorem 6.1 (see [33]) Suppose $\left(\mathrm{H}_{1}\right)$ holds. Then necessary and sufficient conditions for the existence of positive solutions from $C[0,1]$ for the boundary value problem (6.4) are

$$
\begin{aligned}
& 0<\int_{0}^{1} x(1-x) f(x, 1) d x<\infty, \\
& \lim _{x \rightarrow 0} x \int_{x}^{1}(1-s) f(s, 1) d t=0 \quad \text { if } \lim _{x \rightarrow 0} \int_{x}^{1}(1-s) f(s, 1) d s=+\infty, \quad \text { and } \\
& \lim _{x \rightarrow 0}(1-x) \int_{0}^{x} s f(s, 1) d s=0 \quad \text { if } \lim _{x \rightarrow 0} \int_{0}^{x} s f(s, 1) d s=+\infty .
\end{aligned}
$$

Theorem 6.2 (see [33]) Suppose $\left(\mathrm{H}_{1}\right)$ holds. Then a necessary and sufficient condition for the existence of positive solutions from $C^{1}[0,1]$ for the boundary value problem (6.4) is

$$
0<\int_{0}^{1} f(x, x(1-x)) d x<\infty .
$$


Lemma 6.1 (see [34]) Suppose $u \geq 0$ is concave on $[0,1]$. Then

$$
u(t) \geq|u|_{\infty} x(1-x), \quad t \in[0,1] .
$$

By using the idea of the proof in [22] sufficient and necessary conditions for the existence of positive solutions to (6.1) are obtained.

Theorem 6.3 Suppose $\left(\mathrm{H}_{1}\right)$ holds. Then necessary and sufficient conditions for the existence of positive solutions from $C[0,1]$ for the boundary value problem (6.1) are

$$
\begin{aligned}
& 0<\int_{0}^{1} x(1-x) f(x, 1) d x<\infty, \\
& \lim _{x \rightarrow 0} x \int_{x}^{1}(1-s) f(s, 1) d t=0 \quad \text { if } \lim _{x \rightarrow 0} \int_{x}^{1}(1-s) f(s, 1) d s=+\infty, \quad \text { and } \\
& \lim _{x \rightarrow 0}(1-x) \int_{0}^{x} s f(s, 1) d s=0 \quad \text { if } \lim _{x \rightarrow 0} \int_{0}^{x} s f(s, 1) d s=+\infty .
\end{aligned}
$$

Proof Necessity. Suppose that $u_{0}$ is a $C[0,1]$ positive solution to $(6.1)$. Let $v(x)=$ $a\left(\int_{0}^{1} u_{0}(t)^{\gamma} d t\right) u_{0}(x)$, and so $v$ satisfies

$$
\left\{\begin{array}{l}
-v^{\prime \prime}=f\left(x, v / a\left(\int_{0}^{1} u_{0}^{\gamma}(t) d t\right)\right) \stackrel{\text { def }}{=} g(x, v), \quad x \in(0,1) \\
v(0)=v(1)=0 .
\end{array}\right.
$$

It is easy to see that $g(x, v)$ satisfies $\left(\mathrm{H}_{1}\right)$, and Theorem 6.1 implies that

$$
\begin{aligned}
& 0<\int_{0}^{1} x(1-x) g(x, 1) d x<\infty, \\
& \lim _{x \rightarrow 0} x \int_{x}^{1}(1-s) g(s, 1) d s=0 \quad \text { if } \lim _{x \rightarrow 0} \int_{x}^{1}(1-s) g(s, 1) d t=+\infty, \quad \text { and } \\
& \lim _{x \rightarrow 1}(1-x) \int_{0}^{x} s g(s, 1) d s=0 \quad \text { if } \lim _{x \rightarrow 0} \int_{0}^{x} s g(s, 1) d s=+\infty,
\end{aligned}
$$

which is equivalent to (6.5).

Sufficiency. Now we consider

$$
\left\{\begin{array}{l}
-u^{\prime \prime}=\frac{1}{a\left(\int_{\Omega}|u|^{p} d x\right)} f\left(x, u+\frac{1}{n}\right), \quad x \in(0,1), \\
u(0)=u(1)=0 .
\end{array}\right.
$$

Choose $m \geq 2$ such that $m(\mu-\lambda)>1$ and

$$
0<m_{1}=m \frac{\mu-2 \lambda}{\mu-\lambda}<1
$$

Let

$$
h(x)=(1-x) \int_{0}^{x} s f(s, 1) d s+(1-x) \int_{x}^{1}(1-s) f(s, 1) d s, \quad \forall x \in[0,1]
$$


and

$$
H(x)=h(x)^{1 /[m(\mu-\lambda)]}, \quad x \in[0,1],
$$

and, by the proof of [33], define

$$
h_{1}(x)=\int_{0}^{1} G(x, s)(s(1-s))^{\mu} f(s, 1) d s \geq x(1-x)\left|h_{1}\right|_{\infty}, \quad \forall x \in[0,1],
$$

and

$$
H_{1}(x)=\int_{0}^{1} G(x, s)(H(s))^{-\mu} f(s, H(s)) d s+H(x), \quad \forall t \in[0,1],
$$

where

$$
G(x, s)= \begin{cases}s(1-x), & 0 \leq s \leq x \leq 1 \\ x(1-s), & 0 \leq x \leq s \leq 1\end{cases}
$$

Let $H^{*}=\max _{x \in[0,1]} H_{1}(x)$ and choose $K_{1}>0$ large enough such that

$$
K_{1} a_{0} \geq\left(K_{1} H^{*}+1\right)^{\mu}, \quad K_{1} H^{*}>\frac{1}{\delta} .
$$

Let $\beta(x)=K_{1} H_{1}(x), x \in[0,1]$. Inequalities (6.2) and (6.3) guarantee that

$$
\begin{aligned}
f\left(x, \beta(x)+\frac{1}{n}\right) & =f\left(x, \frac{\beta(x)+\frac{1}{n}}{H(x)} H(x)\right) \\
& \leq\left(\frac{\beta(x)+\frac{1}{n}}{H(x)}\right)^{\mu} f(x, H(x)) \\
& \leq\left(K_{1} H^{*}+1\right)^{\mu} H(x)^{-\mu} f(x, H(x)) \\
& \leq a_{0} K_{1} H(x)^{-\mu} f(x, H(x)), \quad x \in(0,1),
\end{aligned}
$$

and so

$$
\left\{\begin{aligned}
-\beta^{\prime \prime}(x) & \geq K_{1} H(x)^{-\mu} f(x, H(x)) \\
& \geq \frac{1}{a_{0}} f\left(x, \beta(x)+\frac{1}{n}\right), \quad x \in(0,1), \\
\beta(0)= & \beta(1)=0 .
\end{aligned}\right.
$$

Let $b_{0}=\sup _{t \in\left[0, \int_{0}^{1} \beta^{\gamma}(t) d t\right]} a(t)$. Let $K_{2}$ be small enough such that

$$
K_{2} h_{1}(x) \leq \beta(x), \quad K_{2} h_{1}(x) \leq 1-\delta, \quad x \in[0,1],
$$

and

$$
b_{0} K_{2}<\delta^{\mu-\lambda}\left(K_{2}\left|h_{1}\right|_{\infty}\right)^{\mu} .
$$


Let $\alpha(x)=K_{2} h_{1}(x), x \in[0,1]$. Inequalities (6.2) and (6.3) guarantee that

$$
\begin{aligned}
f\left(x, \alpha(x)+\frac{1}{n}\right) & \geq \delta^{-\lambda} f\left(x, \delta\left(\alpha(x)+\frac{1}{n}\right)\right) \\
& \geq \delta^{\mu-\lambda}\left(\alpha(x)+\frac{1}{n}\right)^{\mu} f(x, 1) \\
& \geq \delta^{\mu-\lambda}\left(K_{2}\left|h_{1}\right|_{\infty} x(1-x)\right)^{\mu} f(x, 1) \\
& \geq b_{0} K_{2}(x(1-x))^{\mu} f(x, 1), \quad x \in(0,1),
\end{aligned}
$$

and so

$$
\left\{\begin{aligned}
-\alpha^{\prime \prime}(x) & \geq K_{2}(x(1-x))^{\mu} f(x, 1) \\
& \leq \frac{1}{b_{0}} f\left(x, \alpha(x)+\frac{1}{n}\right), \quad x \in(0,1) \\
\alpha(0)= & \alpha(1)=0
\end{aligned}\right.
$$

For $u \in C[0,1]$, from (6.7) and (6.8) we have

$$
\left\{\begin{aligned}
-\beta^{\prime \prime}(x) & \geq \frac{1}{a_{0}} f\left(x, \beta(x)+\frac{1}{n}\right) \\
& \geq \frac{1}{a\left(\int_{0}^{1} \chi^{\gamma}(t, u(t)) d t\right.} f\left(x, \beta(x)+\frac{1}{n}\right), \quad x \in(0,1), \forall u \in C[0,1] \cap C^{2}(0,1), \\
\beta(0)= & \beta(1)=0,
\end{aligned}\right.
$$

and

$$
\left\{\begin{aligned}
-\alpha^{\prime \prime}(x) & \leq \frac{1}{b_{0}} f\left(x, \alpha(x)+\frac{1}{n}\right) \\
& \leq \frac{1}{a\left(\int_{0}^{1} \chi^{\gamma}(t, u(t)) d t\right)} f\left(x, \alpha(x)+\frac{1}{n}\right), \quad x \in(0,1), \forall u \in C[0,1] \cap C^{2}(0,1), \\
\alpha(0)= & \alpha(1)=0 .
\end{aligned}\right.
$$

Consequently, $\alpha$ and $\beta$ are the subsolution and supersolution to (6.6) ${ }_{n}$. By Theorem 2.1, $(6.1)_{n}$ has at least one positive solution $u_{n}$ with $\alpha(x) \leq u_{n}(x) \leq \beta(x), x \in[0,1]$. Moreover, combining (6.2), (6.3), and (6.7), we have

$$
\begin{aligned}
\left|u_{n}^{\prime \prime}(x)\right| & =\frac{1}{a\left(\int_{0}^{1} u_{n}^{\gamma}(x) d x\right)} f\left(x, u_{n}(x)+\frac{1}{n}\right) \\
& \leq \frac{1}{a_{0}} \delta^{\lambda-\mu}\left(\frac{\beta(x)+1}{\alpha(x)+1}\right)^{-\lambda} a_{0} K_{1} H(x)^{-\mu} f(x, H(x)) \\
& \leq \delta^{\lambda-\mu}\left(\frac{\beta(x)+1}{\alpha(x)+1}\right)^{-\lambda} K_{1} H(x)^{-\mu} f(x, H(x)), \quad x \in(0,1),
\end{aligned}
$$

which guarantees that $\left\{u_{n}^{\prime}(x)\right\}$ are uniformly bounded on $\left[\frac{1}{3 k}, 1-\frac{1}{3 k}\right] \subseteq(0,1), k \geq 1$. Therefore, $\left\{u_{n}(x)\right\}$ has a uniformly convergent subsequence on any $\left[\frac{1}{3 k}, 1-\frac{1}{3 k}\right] \subseteq(0,1), k \geq 1$. By the diagonal method there exists a subsequence of $\left\{u_{n}\right\}$ that converges uniformly $u_{0}$ on any $\left[\frac{1}{3 k}, 1-\frac{1}{3 k}\right] \subseteq(0,1), k \geq 1$. Without loss of generality, assume that

$$
\begin{aligned}
& \lim _{n \rightarrow+\infty} u_{n}(x)=u_{0}(x), \quad x \in(0,1), \quad \text { and } \\
& \lim _{n \rightarrow+\infty} u_{n}(x)=u_{0}(x) \quad \text { uniformly } x \in\left[\frac{1}{3 k}, 1-\frac{1}{3 k}\right], k \geq 1 .
\end{aligned}
$$


Obviously, $u_{0}(x)$ is continuous on $(0,1)$, and

$$
\alpha(x) \leq u_{0}(x) \leq \beta(x), \quad x \in[0,1]
$$

Since $\alpha(0)=\beta(0)=\alpha(1)=\beta(1)=0$, we have

$$
\begin{aligned}
& \lim _{x \rightarrow 0} \alpha(x) \leq \lim _{x \rightarrow 0} u_{0}(x) \leq \lim _{x \rightarrow 0} \beta(x) \quad \text { and } \\
& \lim _{x \rightarrow 1} \alpha(x) \leq \lim _{x \rightarrow 1} u_{0}(x) \leq \lim _{x \rightarrow 1} \beta(x),
\end{aligned}
$$

which means that $u_{0}$ is continuous on $[0,1]$ with $u_{0}(0)=u_{0}(1)=0$. The dominated convergence theorem guarantees that

$$
\lim _{n \rightarrow+\infty} \int_{0}^{1} u_{n}^{\gamma}(x) d x=\int_{0}^{1} u_{0}^{\gamma}(x) d x
$$

which, together with the continuity of $a(t)$, means that

$$
\lim _{n \rightarrow+\infty} a\left(\int_{0}^{1} u_{n}^{\gamma}(x) d x\right)=a\left(\int_{0}^{1} u_{0}^{\gamma}(x) d x\right)
$$

Since

$$
\begin{aligned}
u_{n}(x)= & u_{n}\left(\frac{1}{3 k}\right)+u_{n}\left(1-\frac{1}{3 k}\right) \\
& +\frac{1}{a\left(\int_{0}^{1} u_{n}^{\gamma}(x) d x\right)} \int_{\frac{1}{3 k}}^{1-\frac{1}{3 k}} G_{k}(x, s) f\left(s, u_{n}(s)+\frac{1}{n}\right) d x, \quad x \in\left[\frac{1}{3 k}, 1-\frac{1}{3 k}\right],
\end{aligned}
$$

where

$$
G_{k}(x, s)= \begin{cases}\left(s-\frac{1}{3 k}\right)\left(1-\frac{1}{3 k}-x\right), & \frac{1}{3 k} \leq s \leq x \leq 1-\frac{1}{3 k} \\ \left(x-\frac{1}{3 k}\right)\left(1-\frac{1}{3 k}-s\right), & \frac{1}{3 k} \leq x \leq s \leq 1-\frac{1}{3 k}\end{cases}
$$

letting $n \rightarrow+\infty$, we have

$$
\begin{aligned}
u_{0}(x)= & u_{0}\left(\frac{1}{3 k}\right)+u_{0}\left(1-\frac{1}{3 k}\right) \\
& +\frac{1}{a\left(\int_{0}^{1} u_{0}^{\gamma}(x) d x\right)} \int_{\frac{1}{3 k}}^{1-\frac{1}{3 k}} G_{k}(x, s) f\left(s, u_{0}(s)\right) d x, \quad x \in\left[\frac{1}{3 k}, 1-\frac{1}{3 k}\right], k \geq 1 .
\end{aligned}
$$

Differentiating the above equations yields that

$$
-u_{0}^{\prime \prime}(x)=\frac{1}{a\left(\int_{0}^{1} u_{0}^{\gamma}(x) d x\right)} f\left(x, u_{0}(x)\right), \quad x \in\left[\frac{1}{3 k}, 1-\frac{1}{3 k}\right], k \geq 1
$$

which, together with $u_{0}(0)=u_{0}(1)=0$, means that $u_{0} \in C[0,1] \cap C^{2}(0,1)$ and $u_{0}$ is a solution to (6.1). 
Theorem 6.4 Suppose $\left(\mathrm{H}_{1}\right)$ holds. Then a necessary and sufficient condition for the existence of a positive solution from $C^{1}[0,1]$ of $(6.1)$ is

$$
0<\int_{0}^{1} f(x, x(1-x)) d x<\infty .
$$

Proof Necessity. Suppose that $u_{0}$ is a positive solution. Let $v=a\left(\int_{0}^{1} u_{0}^{\gamma}(t) d t\right) u_{0}(t), t \in[0,1]$, and so $v$ satisfies

$$
\left\{\begin{array}{l}
-v^{\prime \prime}=f\left(x, v / a\left(\int_{0}^{1} u_{0}^{\gamma}(t) d t\right)\right) \stackrel{\text { def }}{=} g(x, v), \quad t \in(0,1), \\
v(0)=v(1)=0 .
\end{array}\right.
$$

It is easy to see $g(x, v)$ satisfies $\left(\mathrm{H}_{1}\right)$, and Theorem 6.2 implies that

$$
0<\int_{0}^{1} g(x, x(1-x)) d x<\infty
$$

which is equivalent to (6.9).

Sufficiency. Let

$$
h(x)=(1-x) \int_{0}^{x} s f(s, s(1-s)) d s+(1-x) \int_{x}^{1}(1-s) f(s, s(1-s)) d s, \quad \forall x \in[0,1] .
$$

By Lemma 6.1 we can see that $h(x) \geq x(1-x)|h|_{\infty}$. From (6.9) we know that there exists $N>0$ such that $\int_{0}^{1} s(1-s) f(s, 1-s) d s \leq N$. Then

$$
a_{1} x(1-x) \leq h(x) \leq a_{2} x(1-x), \quad x \in[0,1],
$$

where $a_{1}=\min \{1,\|h\|\}, a_{2}=\max \{1, N\}$.

Let $k_{2}>0$ large enough such that

$$
\frac{1}{a_{0}} a_{2}^{\mu} k_{2}^{\mu-1} \delta^{\lambda-\mu} \leq 1, \quad a_{1} k_{2}>1 .
$$

Inequalities (6.2) and (6.3) imply that

$$
\begin{aligned}
\frac{1}{a_{0}} f(x, \beta(x)) & =\frac{1}{a_{0}} f\left(x, k_{2} h(x)\right) \\
& =\frac{1}{a_{0}} f\left(x, \frac{k_{2} h(x)}{\delta x(1-x)} \delta x(1-x)\right) \\
& \leq \frac{1}{a_{0}}\left(\frac{k_{2} h(x)}{\delta x(1-x)}\right)^{\mu} \delta^{\lambda} f(x, x(1-x)) \\
& \leq \frac{1}{a_{0}} a_{2}^{\mu} k_{2}^{\mu-1} \delta^{\lambda-\mu} k_{2} f(x, x(1-x)) \\
& \leq k_{2} f(x, x(1-x)), \quad x \in(0,1) .
\end{aligned}
$$

Let $k_{1}<k_{2}$ be small enough such that

$$
k_{1}^{\mu-1} \delta^{\lambda-\mu} a_{1}^{\mu} \frac{1}{a\left(\int_{0}^{1}\left(k_{2} h(x)\right)^{\gamma} d x\right)} \geq 1, \quad k_{1} h(t) \leq 1 .
$$


Inequalities (6.2) and (6.3) imply that

$$
\begin{aligned}
\frac{1}{a\left(\int_{0}^{1}\left(k_{2} h(x)\right)^{\gamma} d x\right)} f(x, \alpha(x)) & =\frac{1}{a\left(\int_{0}^{1}\left(k_{2} h(x)\right)^{\gamma} d x\right)} f\left(x, k_{1} h(x)\right) \\
& =\frac{1}{a\left(\int_{0}^{1}\left(k_{2} h(x)\right)^{\gamma} d x\right)} f\left(x, \frac{k_{1} h(x)}{\delta x(1-x)} \delta x(1-x)\right) \\
& \geq \frac{1}{a\left(\int_{0}^{1}\left(k_{2} h(x)\right)^{\gamma} d x\right)}\left(\frac{k_{1} h(x)}{\delta x(1-x)}\right)^{\mu} \delta^{\lambda} f(x, x(1-x)) \\
& \geq \frac{1}{a\left(\int_{0}^{1}\left(k_{2} h(x)\right)^{\gamma} d x\right)} a_{1}^{\mu} k_{1}^{\mu-1} \delta^{\lambda-\mu} k_{1} f(x, x(1-x)) \\
& \geq k_{1} f(x, x(1-x)), \quad x \in(0,1) .
\end{aligned}
$$

Consequently, for $u \in C^{1}[0,1],(6.10)$ and (6.11) guarantee that

$$
\left\{\begin{aligned}
-\beta^{\prime \prime}(x) & =-\left(k_{2} h(x)\right)^{\prime \prime} \\
& =k_{2} f(x, x(1-x)) \\
& \geq \frac{1}{a_{0}} f(x, \beta(x)) \\
& \geq \frac{1}{a\left(\int_{0}^{1} \chi(x, u(x))^{\gamma} d x\right)} f(x, \beta(x)), \quad x \in(0,1), \forall u \in C^{1}[0,1] \cap C^{2}(0,1), \\
\beta(0)= & \beta(0)=0,
\end{aligned}\right.
$$

and

$$
\left\{\begin{aligned}
-\alpha^{\prime \prime}(x) & =-\left(k_{1} h(x)\right)^{\prime \prime} \\
& =k_{1} f(x, x(1-x)) \\
& \leq \frac{1}{a\left(\int_{0}^{1} \beta(x)^{\gamma} d x\right)} f(x, \alpha(x)) \\
& \leq \frac{1}{a\left(\int_{0}^{1} \chi(x, u(x))^{\gamma} d x\right)} f(x, \alpha(x)), \quad x \in(0,1), \forall u \in C^{1}[0,1] \cap C^{2}(0,1), \\
\alpha(0)= & \alpha(0)=0 .
\end{aligned}\right.
$$

Moreover, for $\alpha(x) \leq u \leq v(x)$, choose $c>0$ large enough such that

$$
\frac{c u}{k_{2} x(1-x)} \geq \frac{1}{\delta} \quad \text { and } \quad \frac{k_{2}}{c} \leq \delta, \quad x \in(0,1) .
$$

So, from (6.2) and (6.3) we have

$$
\begin{aligned}
0 & \leq f(x, u) \\
& =f\left(x, \frac{k_{2}}{c} \frac{c u}{k_{2} x(1-x)} x(1-x)\right) \\
& \leq\left(\frac{k_{2}}{c}\right)^{\lambda}\left(\frac{c u}{k_{2} x(1-x)}\right)^{\mu} f(x, x(1-x)) \\
& \leq\left(\frac{k_{2}}{c}\right)^{\lambda}\left(c a_{2}\right)^{\mu} f(x, x(1-x)),
\end{aligned}
$$

which, together with (6.9), guarantees that

$$
\int_{0}^{1}|f(x, u)| d x \leq\left(\frac{k_{2}}{c}\right)^{\lambda}\left(c a_{2}\right)^{\mu} \int_{0}^{1} f(x, x(1-x)) d x<+\infty, \quad \forall \alpha(x) \leq u \leq v(x) .
$$


By Theorem 2.1, (6.1) has at least one positive solution $u \in C^{1}[0,1]$ with $\alpha(x) \leq u(x) \leq \beta(x)$, $x \in[0,1]$.

\section{Competing interests}

The authors declare that there is no conflict of interests regarding the publication of this paper.

\section{Authors' contributions}

Both authors contributed equally in this article. They read and approved the final manuscript.

\section{Acknowledgements}

This research is supported by Young Award of Shandong Province (ZR2013AQ008) and the Fund of Science and Technology Plan of Shandong Province (2014GGH201010).

Received: 23 June 2016 Accepted: 31 August 2016 Published online: 13 September 2016

\section{References}

1. Chipot, M, Lovat, B: Some remarks on nonlocal elliptic and parabolic problems. Nonlinear Anal. 30, 4619-4627 (1997)

2. Corrêa, FJSA: On positive solutions of nonlocal and nonvariational elliptic problems. Nonlinear Anal. 59, 1147-1155 (2004)

3. Corrêa, FJSA, Menezes, SDB, Ferreira, J: On a class of problems involving a nonlocal operator. Appl. Math. Comput. $147,475-489$ (2004)

4. Chipot, M, Corrêa, FJSA: Boundary layer solutions to functional elliptic equations. Bull. Braz. Math. Soc. 40(3), 381-393 (2009)

5. Chipot, M, Roy, P: Existence results for some functional elliptic equations. Differ. Integral Equ. 27, 289-300 (2014)

6. Alves, CO, Corrêa, FJSA: On existence of solutions for a class of problems involving a nonlinear operator. Commun. Appl. Nonlinear Anal. 8(2), 43-56 (2001)

7. Alves, CO, Corrêa, FJSA, Figueiredo, GM: On a class of nonlocal elliptic problems with critical growth. Differ. Equ. Appl. 2, 409-417 (2010)

8. Corrêa, FJSA, Figueiredo, GM: On an elliptic equation of $p$-Kirchhoff type via variational methods. Bull. Aust. Math. Soc 74, 263-277 (2006)

9. Figueiredo, GM: Existence of positive solution for a Kirchhoff problem type with critical growth via truncation argument. J. Math. Anal. Appl. 401(2), 324-353 (1974)

10. Li, Y, Li, F, Shi, J: Existence of a positive solution to Kirchhoff type problems without compactness conditions. J. Differ. Equ. 253, 2285-2294 (2012)

11. Ma, TF: Remarks on an elliptic equation of Kirchhoff type. Nonlinear Anal. 63(5-7), 1967-1977 (2005)

12. Perera, K, Zhang, Z: Nontrivial solutions of Kirchhoff-type problems via the Yang index. J. Differ. Equ. 221(1), 246-255 (2006)

13. Zhang, Z, Perera, K: Sign changing solutions of Kirchhoff type problems via invariant sets of descent flow. J. Math. Anal. Appl. 317(2), 456-463 (2006)

14. Chen, $C, K u o, Y, W u, T$ : The Nehari manifold for a Kirchhoff type problem involving sign-changing weight functions. J. Differ. Equ. 250, 1876-1908 (2011)

15. Adimurthy, Pacella, F, Yadava, L: On the number of positive solutions of some semilinear Dirichlet problems in a ball. Differ. Integral Equ. 10, 1157-1170 (1997)

16. Ambrosetti, A, Azorero, JG, Peral, I: Multiplicity results for some nonlinear elliptic equations. J. Funct. Anal. 137, 219-242 (1996)

17. Damascelli, L, Grossi, M, Pacella, F: Qualitative properties of positive solutions of semilinear elliptic equations in symmetric domains via the maximum principle. Ann. Inst. Henri Poincaré, Anal. Non Linéaire 16, 631-652 (1999)

18. Korman, P: On uniqueness of positive solutions for a class of semilinear equations. Discrete Contin. Dyn. Syst. 8 865-871 (2002)

19. Lions, PL: On the existence of positive solutions of semilinear elliptic equations. SIAM Rev. 24(4), 441-467 (1982)

20. Ouyang, T, Shi, J: Exact multiplicity of positive solutions for a class of semilinear problem II. J. Differ. Equ. 158, 94-151 (1999)

21. Shi, J, Yao, M: On a singular nonlinear semilinear elliptic problem. Proc. R. Soc. Edinb., Sect. A 128, 1389-1401 (1995)

22. Tang, M: Exact multiplicity for semilinear elliptic Dirichlet problems involving concave and convex nonlinearities. Proc. R. Soc. Edinb., Sect. A 133, 705-717 (2003)

23. Ambrosetti, A, Brezis, H, Cerami, G: Combined effects of concave and convex nonlinearities in some elliptic problems, J. Funct. Anal. 122, 519-543 (1994)

24. Zhang, Z: A remark on the existence of entire solutions of a singular semilinear elliptic problem. J. Math. Anal. Appl. 215(2), 579-582 (1997)

25. Zhang, Z: Positive solutions for Dirichlet problems associated to semilinear elliptic equations with singular nonlinearity. Nonlinear Anal., Theory Methods Appl. 21(3), 181-190 (1993)

26. Alves, CO, Covei, DP: Existence of solution for a class of nonlocal elliptic problem via sub-supersolution method. Nonlinear Anal., Real World Appl. 23, 1-8 (2015)

27. Alves, CO, Delgado, M, Souto, MAS, Suárez, A: Existence of positive solution of a nonlocal logistic population model. Z. Angew. Math. Phys. 66(3), 943-953 (2015)

28. De Coster, C, Henrard, M: Existence and localization of solution for second order elliptic BVP in presence of lower and upper solutions without any order. J. Differ. Equ. 145, 420-452 (1998)

29. Yan, B, Wang, D: The multiplicity of positive solutions for a class of nonlocal elliptic problem. J. Math. Anal. Appl. 442(1), 72-102 (2016) 
30. Berger, MS: Nonlinearity and Functional Analysis. Lectures on Nonlinear Problems in Mathematical Analysis. Academic Press, San Diego (1977)

31. Rabinowitz, P: Some global results for nonlinear eigenvalue problems. J. Funct. Anal. 7, 487-513 (1971)

32. Gui, C, Lin, F: Regularity of an elliptic problem with a singular nonlinearity. Proc. R. Soc. Edinb., Sect. A 123, 1021-1029 (1993)

33. Wei, Z: Positive solutions of nonresonant singular boundary value problems for second order differential equation system. Nagoya Math. Anal. J. 162, 127-148 (2001)

34. Agarwal, RP, O'Regan, D: A survey of recent results for initial and boundary value problems singular in the dependent variable. In: Handbook of Differential Equations: Ordinary Differential Equations, vol. 1, pp. 1-68. Elsevier, Amsterdam (2000)

Submit your manuscript to a SpringerOpen ${ }^{\circ}$ journal and benefit from:

- Convenient online submission

- Rigorous peer review

- Immediate publication on acceptance

- Open access: articles freely available online

- High visibility within the field

- Retaining the copyright to your article 